TRANSACTIONS OF THE

AMERICAN MATHEMATICAL SOCIETY

Volume 351, Number 6, Pages 2375-2406

S 0002-9947(99)02445-9

Article electronically published on February 15, 1999

\title{
THE VISCOUS CAHN-HILLIARD EQUATION: MORSE DECOMPOSITION AND STRUCTURE OF THE GLOBAL ATTRACTOR
}

\author{
M. GRINFELD AND A. NOVICK-COHEN
}

\begin{abstract}
In this paper we establish a Morse decomposition of the stationary solutions of the one-dimensional viscous Cahn-Hilliard equation by explicit energy calculations. Strong non-degeneracy of the stationary solutions is proven away from turning points and points of bifurcation from the homogeneous state and the dimension of the unstable manifold is calculated for all stationary states. In the unstable case, the flow on the global attractor is shown to be semi-conjugate to the flow on the global attractor of the Chaffee-Infante equation, and in the metastable case close to the nonlocal reaction-diffusion limit, a partial description of the structure of the global attractor is obtained by connection matrix arguments, employing a partial energy ordering and the existence of a weak lap number principle.
\end{abstract}

\section{INTRODUCTION}

In this paper, we consider the viscous Cahn-Hilliard equation

$$
(1-\alpha) \eta_{t}=\Delta\left[f(\eta)-\epsilon^{2} \Delta \eta+\alpha \eta_{t}\right],
$$

where $f(\eta)=F^{\prime}(\eta)$ and where $F$ is a double well free energy density on a bounded domain $\Omega$ in $\mathbf{R}^{n}, n=1,2$ or 3 , though we shall focus primarily on the case $n=$ 1. Here $\epsilon$ measures the range of the intermolecular forces, and $\eta$ represents, for example, the concentration of one of two components of a binary viscous liquid system. The viscous Cahn-Hilliard equation has been proposed as a model for phase separation in glass and polymer systems where intermolecular friction forces may be expected to be of importance. A derivation of this equation is given in [39]. For a review, see [41]. The boundary conditions are typically taken to be

$$
\nabla \eta \cdot \mathbf{n}=0 \text { and } \mathbf{J} \cdot \mathbf{n}=0
$$

where

$$
\mathbf{J}=-\nabla\left[f(\eta)-\epsilon^{2} \Delta \eta\right]
$$

Note that if we take $\alpha=0$, the Cahn-Hilliard equation [12],

$$
\eta_{t}=\Delta\left[f(\eta)-\epsilon^{2} \Delta \eta\right]
$$

is obtained. It is a well accepted macroscopic field-theoretical model of processes such as phase separation in a binary alloy. Here also it is appropriate to consider the boundary conditions (1.2). On the other hand, taking $\alpha=1$ in the viscous

Received by the editors September 24, 1996.

1991 Mathematics Subject Classification. Primary 35K22, 35K30, 58F12, 58F39.

(C)1999 American Mathematical Society 
Cahn-Hilliard equation, a simple calculation shows that the result is the nonlocal reaction-diffusion equation [46]

$$
\eta_{t}=\epsilon^{2} \Delta \eta-f(\eta)+\frac{1}{|\Omega|} \int_{\Omega} f(\eta) d x,
$$

which is a nonlocal second order parabolic equation. Here $\eta$ is taken to satisfy Neumann boundary conditions.

A different way of deriving equation (1.1) and (1.4) has been suggested by Fife [21]. One can take the Ginzburg-Landau free energy functional

$$
\mathcal{F}(\eta)=\int_{\Omega}\left\{F(\eta)+\frac{\epsilon^{2}}{2}|\nabla \eta|^{2}\right\} d x
$$

and look for constrained (mass-conserving) gradient flows of this functional. The equation one obtains depends on the choice of definition of the gradient flow. Gradient flow in the sense of the $H^{-1}(\Omega)$ inner product yields the Cahn-Hilliard equation, while gradient flow in $L^{2}(\Omega)$ inner product leads to the nonlocal reaction-diffusion equation; a weighted average of these two inner products gives (1.1).

It is not hard to show using the ideas of $[29,17]$ (see $\S 3$ ) that the viscous CahnHilliard possesses a global attractor which is compact, connected and consists of equilibria and orbits connecting them. In one space dimension the equilibria are isolated [37] and the global attractor is finite-dimensional; in higher dimension this is not in general the case. For the Cahn-Hilliard equation one knows, moreover, that there exist inertial manifolds [37] and inertial sets [15]. By definition, the inertial manifolds and inertial sets are finite dimensional, exponentially attracting, and contain the global attractor.

As far as dependence of the attractors on $\alpha$ is concerned, the following theorem can be proved using the ideas of [29] and [17]. Let us denote by $\mathcal{A}_{\alpha}^{m}$ the attractor of the viscous Cahn-Hilliard equation in one space dimension for a given value of $m$ on a given interval $\Omega=[0, L]$, where the dependence of the global attractor resulting from the mass constraint has been indicated explicitly.

Theorem 1.1. The sets $\left\{\mathcal{A}_{\alpha}^{m}\right\}$ are Hausdorff-continuous with respect to $\alpha$ and $m$ at $\alpha=1$ for $m \in(-1,1)$.

We only indicate here the semigroup setting for this theorem; the detailed arguments can be found in $\S 3$. Our initial discussion is general and not restricted to $n=1$. The most useful way of writing (1.1) in this context is:

$$
(1-\alpha) \eta_{t}=\Delta w, \quad \alpha \eta_{t}=\epsilon^{2} \Delta \eta-f(\eta)+w,
$$

in $\Omega$, with boundary conditions

$$
\nabla \eta \cdot \mathbf{n}=\nabla w \cdot \mathbf{n}=0 \text { on } \partial \Omega .
$$

Now let

$$
\dot{L}^{2}(\Omega)=\left\{f \in L^{2}(\Omega), \int_{\Omega} f(x) d x=0\right\} .
$$

Using the fact that

$$
\int_{\Omega} w d x=\int_{\Omega} f(\eta) d x
$$


and incorporating the constraint

$$
\int_{\Omega} \eta(x, t) d x=\int_{\Omega} \eta(x, 0) d x=m
$$

by setting $v=\eta-m$, the equation

$$
v_{t}=\mathcal{B}_{\alpha}^{-1}\left(\epsilon^{2} \Delta v-f(v+m)+\frac{1}{|\Omega|} \int_{\Omega} f(v+m) d x\right)
$$

is obtained, where

$$
\mathcal{B}_{\alpha}=\alpha I+(1-\alpha) \Delta^{-1} .
$$

Here $\Delta^{-1}$ is the Green's function of $-\Delta$ on $\Omega$ with Neumann boundary conditions acting on functions in $\dot{L}^{2}(\Omega) . \mathcal{B}_{\alpha}$ is an invertible operator from $H^{2}(\Omega) \cap \dot{L}^{2}(\Omega)$ into $\dot{L}^{2}(\Omega)$ for $\alpha \in[0,1]$. We shall restrict our attention to the case $m \in[0,1]$, since the case $m \in[-1,0]$ follows by symmetry and the case $m \in[-\infty, \infty] \backslash[-1,1]$ corresponds to $m$ in the stable regime where the structure of the attractor is trivial.

For all $m \in[0,1]$ and for $\alpha \in[0,1],(1.6)$ generates a semigroup $T_{\alpha}^{m}(t)$ on the space $X=H^{1}(\Omega) \cap \dot{L}^{2}(\Omega)$. It is well known that, for all $\alpha \in[0,1]$, the viscous Cahn-Hilliard equation (1.1) conserves mass. In the process of the proof of Theorem 1.1 it is also shown that the above semigroups have nice joint continuity properties in terms of $\alpha$ and $m$ in the sense of (H7bis) $\epsilon$ of Theorem 4.10.8 of [29]. This fact is used in the sequel.

Furthermore, we have the following lemma.

Lemma 1.2. The dimensions of the unstable manifolds of equilibria are independent of $\alpha$.

Proof. Note from (1.6) that the eigenvalue problem associated with linearizing the viscous Cahn-Hilliard equation around a stationary solution $u$ is

$$
\rho \mathcal{B}_{\alpha} \phi+\mathcal{H} \phi=0, \phi \in \dot{L}^{2},
$$

where

$$
\mathcal{H} \phi=\epsilon^{2} \Delta \phi-f^{\prime}(u) \phi+\frac{1}{|\Omega|} \int_{\Omega} f^{\prime}(u) \phi d x .
$$

Assume that $\phi$ is a zero-eigenfunction of $\mathcal{H}$; boundedness of $\mathcal{B}_{\alpha}^{-1}$ implies that $\phi$ is also a zero-eigenfunction of $\mathcal{B}_{\alpha}^{-1} \mathcal{H}$ for all $\alpha \in[0,1]$. Similarly, if $\phi$ is a zeroeigenfunction of $\mathcal{B}_{\alpha}^{-1} \mathcal{H}$ for some $\alpha \in[0,1]$, it is also a zero-eigenfunction of $\mathcal{H}$. Hence the dimensionality of the zero eigenspace is the same for all $\alpha \in[0,1]$.

Now set $L_{P}^{2}(\Omega)=\dot{L}^{2}(\Omega) \backslash\{$ span of zero eigenfunctions $\}$, and consider the operator equation

$$
\rho \mathcal{G} \mathcal{B}_{\alpha} \phi+\phi=0
$$

for $\phi \in L_{P}^{2}(\Omega)$, where $\mathcal{G}=\mathcal{H}^{-1}$. Since $\mathcal{G}$ and $\mathcal{B}_{\alpha}$ are self-adjoint bounded operators on $L_{P}^{2}(\Omega), \mathcal{G}$ is compact, and $\left(\mathcal{B}_{\alpha} \psi, \psi\right)>0$ for all nonzero $\psi \in L_{P}^{2}(\Omega)$ and all $\alpha \in[0,1]$, the results of the appendix of [6] apply. Hence we conclude that the number of unstable eigenvalues is identical for all $\alpha \in[0,1]$. Combining this with the control on the number of zero-eigenfunctions described above, the lemma is proved. 
The object of this paper is to present results contributing towards a partial picture of the variation in the structure of the attractor (in one space dimension) for the viscous Cahn-Hilliard equation as the mass constraint and homotopy parameter are varied. The new information obtained here is due to a partial ordering of the stationary states according to their free energies. A fuller description of the attractor is presented for $\alpha$ close to 1, that is, close to the nonlocal reactiondiffusion equation limit; this is possible thanks to lap number type principles given in Lemma 1.3 and Theorem 1.4 below coupled with semi-conjugacy and connection matrix type arguments $[35,30]$. In the final section we present some remarks on the difficulties which arise when attempting a fuller description for the whole homotopy of the viscous Cahn-Hilliard equation. We note that the situation in more than one dimension is much more complicated. While there is considerable information about long time asymptotics [3, 4], the structure of the equilibria and their connecting orbits is as present unclear, although some partial results on existence, (in)stability and minimizing properties of specific types of equilibria are known [28], [36] [48] [50].

From now on we take $n=1$, so that $\Omega$ is a bounded interval, and consider the rescaled problem

$$
\begin{gathered}
(1-\alpha) \eta_{t}=\left[f(\eta)-\eta_{x x}+\alpha \eta_{t}\right]_{x x}, \quad x \in(0, L), \\
\eta_{x}(0)=\eta_{x}(L)=0, \quad \eta_{x x x}(0)=\eta_{x x x}(L)=0 .
\end{gathered}
$$

Now we utilize the fact that the nonlocal reaction-diffusion equation is a second order equation. In fact the crucial statement is:

Lemma 1.3. If $u(x, t)$ is a solution of the nonlocal reaction-diffusion equation, then its lap number, $l(u)$ is nonincreasing with time.

Proof. Note that equation satisfied by $u=\eta_{x}$ is a local second order parabolic equation, so that the arguments of Matano [34] apply.

Lemma 1.3 shows that the nonlocal reaction-diffusion equation has what we shall call the strong lap number property. By the weak lap number property we would mean the following situation: let $\phi, \psi$ be two equilibria. Then if $C(\phi, \psi) \neq \emptyset$, that is, if there are connections from $\phi$ to $\psi$, then necessarily $l(\phi) \geq l(\psi)$. Clearly, the strong lap number property implies the weak one.

Remark. It was demonstrated in [8], for the Cahn-Hilliard equation, that even though a weak lap number principle may hold, the strong lap number principle fails to hold along certain connections. On the other hand, in the context of delay differential equations [33] a strong lap number property may hold on the attractor only.

Theorem 1.4. The semigroups $T_{\alpha}^{m}(t)$ have the weak lap number property for $\alpha$ close to 1 .

This theorem is a corollary of the following general result:

Proposition 1.5. Under the conditions of Theorem 1.1, the following is true: If $\mathcal{A}_{1}^{m}$ admits an attractor-repellor pair $A_{1}^{m}, R_{1}^{m}$, then for $\alpha$ sufficiently close to $1, \mathcal{A}_{\alpha}^{m}$ admits attractor-repellor pairs $A_{\alpha}^{m}, R_{\alpha}^{m}$, such that the families of sets $\left\{A_{\alpha}^{m}\right\},\left\{R_{\alpha}^{m}\right\}$ are Hausdorff continuous at $\alpha=1$. Furthermore, if $C\left(R_{1}^{m}, A_{1}^{m}\right)$ is nonempty, so is $C\left(R_{\alpha}^{m}, A_{\alpha}^{m}\right)$ for $\alpha$ sufficiently close to 1 . 
The proof follows standard arguments in Conley index theory; see [22].

Proof of Theorem 1.4. Using the above proposition, we need only notice that if $K$ is the largest lap number of any equilibrium in $\mathcal{A}_{1}^{m}$, we can take $A_{1}^{m}$ to be the set of equilibria of lap numbers not exceeding $k$ for any $k<K$ and all connections between such equilibria, with $R_{1}^{m}$ being the dual repellor. Then from the proposition and Theorem 1.1 it follows that for $\alpha$ sufficiently close to 1 all equilibria in $A_{1}^{m}$ are contained in $A_{\alpha}^{m}$, and, furthermore, that there are no connections from these equilibria to the equilibria with lap numbers higher than $k$ contained in $R_{\alpha}^{m}$. Since $k$ is arbitrary, the theorem is proved.

In the one-dimensional case, with the free energy density chosen to be quartic, a full description of the stationary states for the viscous Cahn-Hilliard equation has been given in [27]. The main result there is the following:

Theorem 1.6. 1. For any $m, 0<m<1 / \sqrt{5}$, for any $L \leq \pi / \sqrt{1-3 m^{2}}$ there are no nontrivial stationary monotone increasing solutions for the viscous Cahn-Hilliard equation and for any $L>\pi / \sqrt{1-3 m^{2}}$ there is a unique nontrivial stationary monotone increasing solution for the viscous Cahn-Hilliard equation.

2. Similarly, for any $m, 1 / \sqrt{5}<m<1 / \sqrt{3}$, there exists an $L^{\gamma}(m) \geq \pi$ such that for $L<L^{\gamma}(m)$ there are no nontrivial stationary solutions of the viscous Cahn-Hilliard equation, for $L^{\gamma}(m)<L<\pi / \sqrt{1-3 m^{2}}$ there are precisely two nontrivial stationary monotone increasing solutions for the viscous Cahn-Hilliard equation, and for $L \geq \pi / \sqrt{1-3 m^{2}}$ there is precisely one such solution.

3. Lastly, for any $1 / \sqrt{3}<m<1$, there is an $L^{\gamma}(m)$, such that for any $L<$ $L^{\gamma}(m)$ there are no nontrivial stationary solutions to the viscous CahnHilliard equation, while for any $L>L^{\gamma}(m)$ there are precisely two stationary monotone increasing solutions for the viscous Cahn-Hilliard equation. Furthermore, $L^{\gamma}(m) \rightarrow \infty$ as $m \rightarrow 1$.

Henceforth, we distinguish between three cases, the unstable case: $0 \leq m<\frac{1}{\sqrt{5}}$, the transitional [38] case: $\frac{1}{\sqrt{5}} \leq m<\frac{1}{\sqrt{3}}$, and the metastable case: $\frac{1}{\sqrt{3}} \leq m<1$.

The methods of analyzing intersections of level curves, which were used in [27] and [42] are also useful for deriving energy estimates and in understanding the structure of the stationary states. For this reason we point out that all monotone stationary solutions may be parametrized by two parameters, $p$ and $a$, restricted to lie in an admissible region $\Sigma$. Symmetry considerations leads us to consider the subregion of $\Sigma$ which corresponds to $m \geq 0$. This subregion will be denoted by $\Sigma^{+}$ and is given by $\Sigma^{+}=\Sigma \cap\{a \geq 0\}$. The region $\Sigma^{+}$can be characterized as follows $[27],[42]$ :

Lemma 1.7. The boundaries of $\Sigma^{+}$are the curves (parametrized by $p$ ):

$$
\begin{gathered}
\sigma_{0}=\left\{(p, 0), 0<p<\frac{1}{4}\right\} ; \\
\sigma_{1}=\left\{\left(p, a_{1}(p)\right)=\left(p, \sqrt{\frac{1}{27}-\frac{4 p}{3}+\frac{1}{27}(12 p+1)^{3 / 2}}\right),-\frac{1}{12}<p \leq \frac{1}{4}\right\} ;
\end{gathered}
$$




$$
\sigma_{2}=\left\{\left(p, a_{2}(p)\right)=\left(p, \sqrt{\frac{1}{27}-\frac{4 p}{3}-\frac{1}{27}(12 p+1)^{3 / 2}}\right),-\frac{1}{12}<p \leq 0\right\} .
$$

In terms of the level sets of the average concentration $\mathcal{C}_{m}$, we have [42],[27]:

Lemma 1.8. For $\widehat{m} \in(0,1 / \sqrt{3})$, the level set $\mathcal{C}_{\widehat{m}}$ consists of a unique curve connecting the point $(1 / 4,0)$ with a point lying on the curve $\sigma_{2}$, and for $\widehat{m} \in(1 / \sqrt{3}, 1)$, the level set $\mathcal{C}_{\widehat{m}}$ consists of a unique curve connecting the point $(1 / 4,0)$ with a point lying on the curve $\sigma_{1}$.

The structure of this paper is as follows. After preliminary observations in $\S 2$, in $\S 3$ Hausdorff-continuity of attractors $\mathcal{A}_{\alpha}^{m}$ at $\alpha=1$ is proven and in $\S 4$ we show how to order stationary solutions by evaluating their free energy and relying heavily on the techniques of [27]. In $\S 6$ nondegeneracy of the stationary states away from turning points and points of bifurcation from the homogeneous state is demonstrated and the dimension of the unstable manifolds is calculated using continuity arguments in $\Sigma^{+}$, connection matrix methods, and a comparison theorem from [7]. Finally, in $\S 7$ we discuss the structure of the attractor, treating both the stable and metastable cases.

\section{Notation}

In this section we introduce a consistent notation for the set of all stationary solutions of the viscous Cahn-Hilliard equation. In particular, for a given value of the mass $m \in[0,1]$ we need to parameterize all branches of stationary solutions.

Note first that, for any $m \in[0,1]$, there always exists the trivial homogeneous solution $u(x) \equiv m$, which we denote here by $M_{h}(m)$. Such solutions correspond to points $(p, a)$ lying on $\sigma_{1} \cup \sigma_{2}$. In order to consider the monotone stationary solutions, we turn to the information given in Theorem 1 of the Introduction. Consider first the unstable case, (i). Here, setting $L_{\min }(m)=\pi / \sqrt{1-3 m^{2}}$, there clearly exists a unique monotone increasing nontrivial solution for any $L$ in the interval $\left(L_{\min }(m), \infty\right)$. Note that $L_{\min }(m)=L_{\sigma_{2}}(m)$, i.e. the value of $L$ assumed at the intersection of the curve $\mathcal{C}_{m}$ with the curve $\sigma_{2}$. Therefore these solutions may be conveniently parametrized by the interval length $L$. For large values of $L$, corresponding to points on the curve $\mathcal{C}_{m}$ lying near $(1 / 4,0)$, these solutions are known to behave like interface solutions [13], i.e., they have no boundary layers and contain a transition layer whose width is $\mathcal{O}(1 / L)$. Thus it is convenient to use for these solutions the notation $M_{i}^{1+}(L, m)$, where the superscript 1 indicates the lap number $l$ of the solution, while the superscript + refers to the fact that these solutions are monotone increasing, and the subscript $i$ refers to the fact that these solutions behave asymptotically like interface solutions. The letter $M$ here is used in this paper to denote the various solutions and sets of solutions, due to the fact that they will be the Morse sets in Morse decompositions to come. Noting that there also exist decreasing as well as increasing solutions, since it is easy to check that if $u=u(x)$ is a stationary solution for a given value of $L$ and $m$, then so is $u=u(-x)$; the monotone decreasing solutions will similarly be parametrized as $M_{i}^{1-}(L, m)$.

Additional nonmonotone branches of solutions may be found by considering the following lemma which follows trivially from the consideration of Neumann boundary conditions $[26]$ : 
Lemma 2.1. There exists a stationary solution of the viscous Cahn-Hilliard equation with lap number $k$ on the interval $[0, L]$ with mean mass $m$ if and only if there exists a monotone stationary solution of the viscous Cahn-Hilliard equation with means mass $m$ on the interval number $[0, L / k]$.

From the above lemma, we may conclude that for $m$ in the unstable region and for any $L>L_{\min }(m)$ there exist $k$ branches of solutions, where

$$
k=\max _{j \geq 1}\left\{\frac{L}{j}>L_{\min }(m)\right\} .
$$

For these branches, we shall employ the notation $M_{i}^{j \pm}(L, m), 1 \leq j \leq k$.

Considering now the metastable region, and referring to Lemma 1.8, it can be shown by asymptotic analysis (see [8]) that monotone solutions corresponding to points lying on $\mathcal{C}_{m}$ lying near $(1 / 4,0)$ behave like interface solutions; i.e., they contain a smooth internal interface of width $\mathcal{O}(\epsilon)$, and monotone solutions corresponding to points on $\mathcal{C}_{m}$ lying close to the curve $\sigma_{1}$ behave like spike solutions; that is, they contain a boundary layer located near either of the two ends of the interval. Nonmonotone interface solutions are obtained by alternately piecing together monotone solutions and their reflections. Nonmonotone spike solutions are obtained similarly, and we remark that the nomenclature spike refers to the fact by piecing together two nonmonotone spike solutions at their boundary layer ends, a solution is obtained which has the appearance of a spike. Returning to Theorem 1.6 and following the notation used there, for $L>L^{\gamma}(m)$ we obtain $k$ branches of both interface and spike solutions, as prescribed by the formula (2.1), which we parametrize as

$$
\left\{M_{i}^{j \pm}(L, m), M_{s}^{j \pm}(L, m), \quad 1 \leq j \leq k\right\},
$$

in addition to the trivial solution $M_{h}(m)$. Here the superscript $j$ implies that the solution is comprised of $j$ monotone solutions, and the superscript $+(-)$ implies that the leftmost monotone solution is increasing (decreasing).

Now, by continuity, for mean masses in the transitional region and for $L>L^{\gamma}(m)$ we again have $k$ branches of interface solutions with $k$ defined as before, which we parametrize as

$$
\left\{M_{i}^{j \pm}(L, m), \quad 1 \leq j \leq k\right\}
$$

in addition to $k_{s}$ spike solutions

$$
\left\{M_{s}^{j \pm}(L, m), \quad 1 \leq j \leq k_{s}\right\}
$$

where

$$
k_{s}=k-k_{0}, \quad \text { for } \quad k_{0}=\max _{j \geq 0}\left\{\frac{L}{j} \geq \frac{\pi}{\sqrt{1-3 m^{2}}}\right\},
$$

as well as the trivial homogeneous solution $M_{h}(m)$.

\section{HausdorfF-CONTINUity of the attractor}

The discussion in this section follows roughly the treatment given in [17] where the properties of the attractor for the viscous Cahn-Hilliard equation with Dirichlet boundary conditions were studied. For the viscous Cahn-Hilliard equation with Dirichlet boundary conditions, the mean mass is not preserved; as the mean mass is conserved under the action of the semigroup of the viscous Cahn-Hilliard equation 
with Neumann boundary conditions, the conclusions given there must be altered accordingly.

In order to prove Hausdorff-continuity and other basic properties of the attractor, it is necessary to discuss the regularity of solutions of the viscous Cahn-Hilliard equation. Let $|\cdot|$ denote the $L^{2}(\Omega)$ norm, $|\cdot|_{s}$ the $H^{s}(\Omega)$ norm, and $\|\cdot\|$ the $H^{1}(\Omega)$ norm. It is easy to see that

$$
\left(B_{\alpha} v_{t}, v_{t}\right)=\mathcal{F}(v) \geq c_{1}\|v+m\|-c_{2}
$$

where $c_{1}$ and $c_{2}$ are positive constants.

Theorem 3.1. For any $\alpha \in[0,1], m \in[0,1]$ and any $v_{0} \in \dot{L}^{2}(\Omega)$, there exists a unique solution $v(t)$ to the viscous Cahn-Hilliard equation (1.6) such that $v \in$ $C\left([0, T] ; \dot{L}^{2}(\Omega)\right) \cap C^{1}\left(0, T ; \dot{H}^{-1}(\Omega)\right)$ for any $T>0$. For all $\alpha \in[0,1]$, there exists an absorbing ball in $\dot{H}^{2}(\Omega)$. If, moreover, $v_{0} \in \dot{H}^{1}(\Omega)$, then $v \in C\left([0, T] ; \dot{H}^{1}(\Omega)\right) \cap$ $C^{1}\left(0, T ; \dot{L}^{2}(\Omega)\right)$ for any $T>0$.

Let $T_{\alpha}\left(t ; u_{0}, m\right)$ denote the solution of (1.6) for given initial conditions and mean mass on the interval $[0, L]$, and let $\partial_{t}, \partial D, D_{m}$ denote its Fréchet derivative with respect to time, initial conditions, and mean mass, respectively. Then for any $m \in[0,1]:$

$$
\begin{gathered}
\left|T_{0}\left(t ; u_{0}, m\right)\right|_{\beta} \leq C_{1}(R, \beta)\left[1+t^{-(\beta-1) / 4}\right] \quad \forall t>0 \quad \forall \beta \in[1,4], \\
\left|T_{\alpha}\left(t ; u_{0}, m\right)\right|_{\beta} \leq C_{1}^{\alpha}(R, \beta)\left[1+t^{-(\beta-1) / 2}\right] \quad \forall t>0 \quad \forall \beta \in[1,2] \quad \forall \alpha \in(0,1], \\
\left|\partial_{t} T_{0}\left(t ; u_{0}, m\right)\right|_{0} \leq C_{2}(R, T)\left[1+t^{-3 / 4}\right] \quad \forall t>0, \\
\left|\partial_{t} T_{\alpha}\left(t ; u_{0}, m\right)\right|_{0} \leq C_{2}^{\alpha}(R, T)\left[1+t^{-1 / 2}\right] \quad \forall t>0 \quad \forall \alpha \in(0,1], \\
\left|\partial_{t} D T_{0}\left(t ; u_{0}, m\right) w\right| \leq C_{3}(R, T) t^{-3 / 4}|| w||, \quad t \in(0, T], \\
\left|\partial_{t} D T_{\alpha}\left(t ; u_{0}, m\right) w\right| \leq C_{3}^{\alpha}(R, T) t^{-1 / 2}|| w|| \quad \forall \alpha \in(0,1], \quad t \in(0, T], \\
|| D T_{0}\left(t ; u_{0}, m\right) w|| \leq C_{4}(R, T)\|w\|, \quad t \in(0, T], \\
\left|\partial_{t} D_{m} T_{0}\left(t ; u_{0}, m\right) \tilde{m}\right| \leq C_{5}(R, T) t^{-3 / 4}|\tilde{m}| \quad \forall t>0, \\
\left|\partial_{t} D_{m} T_{\alpha}\left(t ; u_{0}, m\right) \tilde{m}\right| \leq C_{5}^{\alpha}(R, T) t^{-1 / 2}|\tilde{m}| \leq C_{4}^{\alpha}(R, T)|| w|| \quad \forall t>0 \quad \forall \alpha \in(0,1], \quad t \in(0, T], \\
\left.\left\|D_{m} T_{0}\left(t ; u_{0}, m\right) \tilde{m}\right\| \leq C_{6}(R, T)|\tilde{m}| \quad \forall t>0, \quad t\right], \quad t \in(0, T], \\
|| D_{m} T_{\alpha}\left(t ; u_{0}, m\right) \tilde{m} \| \leq C_{6}^{\alpha}(R, T)|\tilde{m}| \quad \forall t>0 \quad \forall \alpha \in(0,1], \quad t \in(0, T] .
\end{gathered}
$$


Proof. For initial data in $\dot{L}^{2}(\Omega)$, the existence and uniqueness results for the case $\alpha=0$ are proven in [10] and for the case $\alpha \in(0,1]$ they may be proven directly as for the reaction diffusion equation (see [49]). For initial data in $\dot{H}^{1}(\Omega)$, the existence and regularity results cited for the case $\alpha=0$ may be found in [37] or [49], and those cited in the case $\alpha \in(0,1]$ may be proved as in [17] using the (1.6) setting. For the existence of absorbing sets for the case $\alpha=0$ see e.g. [37], and for the case $\alpha \in(0,1]$ the methods of [49] suffice. The inequalities listed above may be proved by combining the methods of $\S 2$ of [17] with the setting of (1.6) and with the existence of absorbing sets cited above.

Corollary 3.2. For each $\alpha \in[0,1]$ and $m \in[0,1], T_{\alpha}^{m}$ is a $C^{1}$-gradient semigroup for which orbits of bounded sets are bounded and which is completely continuous and asymptotically smooth.

Proof. Theorem 3.1 implies that $T_{\alpha}^{m}$ is a strongly continuous $C^{1}$-semigroup. The existence of absorbing sets in $\dot{H}^{2}(\Omega)$, demonstrated in Theorem 3.1, yields that orbits of bounded sets in $\dot{L}^{2}(\Omega)$ are bounded and completely continuous. Corollary 3.2.2 of [29] or Theorem 1.1 of [49] shows that the semigroup is asymptotically smooth. Finally, the existence of the absorbing sets implies that each orbit $T_{\alpha}^{m}$ is precompact and hence, by (3.1), $T_{\alpha}^{m}$ defines a gradient system in the sense of Definition 3.8.1 [29].

Theorem 3.3. Let $\xi, \xi^{\alpha}, \xi^{\alpha+\epsilon} \in B(0, R)$ where $B(0, R)$ is a ball in $\dot{H}^{1}(\Omega), \alpha \in$ $(0,1], m, m^{\alpha}, m^{\alpha+\epsilon} \in[0,1]$, and let $\alpha, \epsilon, \alpha+\epsilon \in[0,1]$. Then there is a constant $C_{1}=C_{1}(\alpha, R)$ such that

$$
\begin{gathered}
\left\|T_{\alpha}\left(t ; \xi^{\alpha}, m^{\alpha}\right)-T_{\alpha+\epsilon}\left(t ; \xi^{\alpha+\epsilon}, m^{\alpha+\epsilon}\right)\right\| \leq C_{1}\left[\left|\xi^{\alpha}-\xi^{\alpha+\epsilon}\right|+\left|m^{\alpha}-m^{\alpha+\epsilon}\right|+\epsilon\right], \\
\left\|D T_{\alpha}(t ; \xi, m) w-D T_{\alpha+\epsilon}(t ; \xi, m) w\right\| \leq C_{1} \epsilon\|w\| .
\end{gathered}
$$

Similarly for $\xi, \xi^{0}, \xi^{\epsilon} \in B(0, R), m, m^{0}, m^{\epsilon} \in[0,1]$, there is a constant $C_{2}=C_{2}(R)$ such that

$$
\begin{gathered}
\left\|T_{0}\left(t ; \xi^{0}, m^{0}\right)-T_{\epsilon}\left(t ; \xi^{\epsilon}, m^{\epsilon}\right)\right\| \leq C_{2}\left(1+t^{-1 / 2}\right)\left[\left|\xi^{0}-\xi^{\epsilon}\right|+\left|m^{0}-m^{\epsilon}\right|+\epsilon\right], \\
\left\|D T^{0}(t ; \xi, m) w-D T^{\epsilon}(t ; \xi, m) w\right\| \leq C_{2}\left(1+t^{-1 / 2}\right) \epsilon\|w\| .
\end{gathered}
$$

Proof. The proof is similar to that given for Theorem 2.3 in [17] and we do not elaborate.

It is proved in $\S 5$ that for all $\alpha \in[0,1]$ and $m \in[0,1]$, away from turning points and points of bifurcation from the homogeneous state, all equilibria are hyperbolic. Since, for a given value of $m, m \in[0,1]$, by Lemmas 4.3 and 4.4 the set of curves in $\Sigma^{+}$corresponding to solutions with constant mean mass $m$ and lap number $k, k \in\{1,2, \ldots\}$, is discrete, the values of $L$ which correspond to turning points or points of bifurcation from the homogeneous state form a discrete set with no accumulation point and may be treated as non-generic. Therefore from the existence of a Lyapunov function, the smoothing properties of Theorem 3.1 and Corollary 3.2, invoking Theorem 3.8.5 of [29], we have that 
Theorem 3.4. For each $\alpha \in[0,1]$ and $m \in[0,1]$ away from turning points and points of bifurcation from the homogeneous state, the semigroup $T_{\alpha}^{m}(t)$ has a compact, connected global attractor $\mathcal{A}_{\alpha}^{m}$ given by

$$
\mathcal{A}_{\alpha}^{m}=\bigcup_{v \in E_{\alpha}^{m}} W(v)
$$

where $W(\cdot)$ denotes the unstable set.

Lastly, Hausdorff-continuity now follows from Theorem 4.10 .8 in [29]; see also [31]:

Theorem 3.5. Let $\alpha_{0} \in(0,1), \quad m_{0} \in(-1,1)$ and suppose that for a given interval length $L, m_{0}$ does not correspond to a turning point or a point of bifurcation from the homogeneous state, then there is a constant $C=C\left(\alpha_{0}\right)>0$ such that for $\epsilon$ and for $\delta$ sufficiently small

$$
d\left(\mathcal{A}_{\alpha_{0}}^{m_{0}}, \mathcal{A}_{\alpha_{0}+\epsilon}^{m_{0}+\delta}\right) \leq C(|\epsilon|+|\delta|) .
$$

Furthermore, there are constants $K_{i}>0, i=1,2$, such that for $\epsilon>0$ and $\epsilon$ and $\delta$ sufficiently small,

$$
d\left(\mathcal{A}_{1}^{m_{0}}, \mathcal{A}_{1-\epsilon}^{m_{0}+\delta}\right) \leq K_{1}(\epsilon+|\delta|)
$$

and

$$
d\left(\mathcal{A}_{0}^{m_{0}}, \mathcal{A}_{\epsilon}^{m_{0}+\delta}\right) \leq K_{2}(\epsilon+|\delta|)
$$

\section{ENERGy SEPARATION}

Taking the (generalized) free energy of a stationary solution $u(x)$ to be

$$
\mathcal{F}(u)=\frac{1}{L} \int_{0}^{L}\left\{\frac{1}{2} u_{x}^{2}+F(u)\right\} d x
$$

we have the following theorem:

Theorem 4.1. For any $m$, let $E(L, i), E(L, s)$ be the free energy of a monotone stationary interface or spike solution, respectively, parametrized by $L$. Then, if $E^{h}=\mathcal{F}(m)$ is the energy of the trivial (homogeneous) solution with mean mass $m$, we have:

(i) For $m \in\left(0, \frac{1}{\sqrt{5}}\right), \quad \lim _{L \rightarrow L_{\min }(m)} E(L, i)=E^{h}(m)$, and for $m \in\left(\frac{1}{\sqrt{5}}, 1\right)$, $\lim _{L \rightarrow L_{\max }(m)} E(L, s)=E^{h}(m)$;

(ii) $\lim _{L \rightarrow \infty} E(L, i)=-1 / 4<E^{h}(m)$;

(iii) $E^{\prime}(L, i)<0, \quad L \neq L^{\gamma}(m)$;

(iv) $E^{\prime}(L, s)<0, \quad L \neq L^{\gamma}(m)$;

(v) For $m \in\left(\frac{1}{\sqrt{5}}, 1\right), \quad E(L, i)<E(L, s), \quad L \in\left(L^{\gamma}(m), L_{\max }(m)\right)$, where $L_{\gamma}(m)$ denotes the value of $L$ assumed at the point of intersection of $\mathcal{C}_{m}$ with the unique curve $\gamma$ in $\Sigma^{+}$of turning points (in [27] the curve $\gamma$ was denoted as $\gamma_{1}$ ), and where

$$
L_{\min }(m)=L_{\sigma_{2}}(m), \quad L_{\max }(m)=\left\{\begin{array}{cc}
L_{\sigma_{2}}(m), & 1 / \sqrt{5} \leq m<1 / \sqrt{3}, \\
\infty, & 1 / \sqrt{3} \leq m \leq 1,
\end{array}\right.
$$




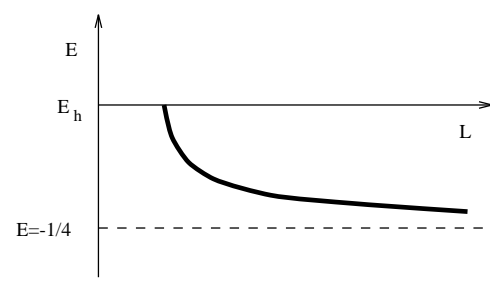

(a)

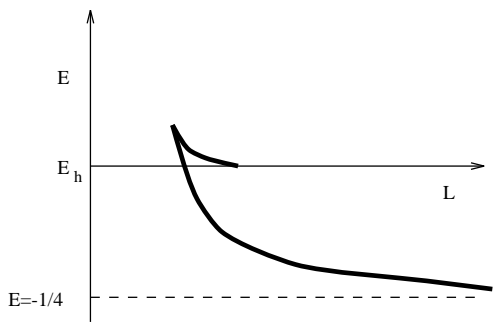

(b)

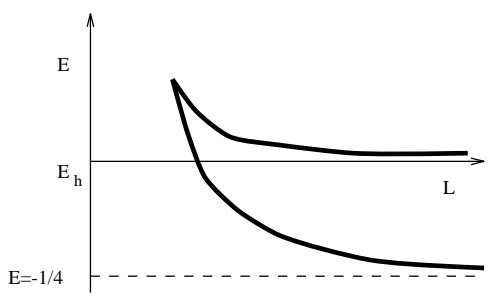

(c)

FiguRE 1. The free energy of the solution branches: (a) the unstable case; (b) the transitional case; (c) the metastable case.

where $L_{\sigma_{2}}(m)=\pi / \sqrt{1-3 m^{2}}$ denotes the value of $L$ assumed at the point of intersection of the curve $\mathcal{C}_{m}$ with the curve $\sigma_{2}$.

Remark. We note that numerically this result was partially verified in [38] and [16]; see Figure 1 as well as the pictures in [6].

The proof relies heavily on the notation, methods, and results of [27]; see also $[26,42]$. For brevity, we summarize in the two theorems which follow the results from the above mentioned papers which will be employed in the proof of Theorem 4.1 .

The first theorem which we present contains what we know about the geometry of $\Sigma^{+}$and the level sets $\mathcal{C}_{L}$ and $\mathcal{C}_{m}$,

Theorem 4.2. Along the boundary of $\Sigma^{+}, \Delta=0$, and within the interior of $\Sigma^{+}$, $\Delta<0$ where

$$
\Delta=-64 p^{3}+32 p^{2}+4\left(18 a^{2}-1\right) p+27 a^{4}-2 a^{2} .
$$

Along $\sigma_{1}$,

$$
L=\infty \text { and } m=\frac{1}{\sqrt{3}}(1+\sqrt{1+12 p})^{1 / 2},
$$

and along $\sigma_{2}$,

$$
L=\pi(1+12 p)^{-1 / 4} \text { and } m=\frac{1}{\sqrt{3}}(1-\sqrt{1+12 p})^{1 / 2} .
$$


These values of $L$ and $m$ correspond to points of bifurcation of monotone solutions from the homogeneous state.

For any $L \in(\pi, \infty)$, there exists a unique level curve $\mathcal{C}_{L}$ connecting a point on $\sigma_{2}$ with a point on $\sigma_{0}$. Moreover,

$$
L_{p}(p, a)>0, \quad L_{a}(p, a)>0, \quad(p, a) \in \Sigma^{+} .
$$

Hence $\mathcal{C}_{L}$ may be parametrized as a unique monotone decreasing curve $a^{L}=a^{L}(p)$.

For any $m \in(0,1)$, there exists a unique level curve in $\Sigma^{+}$which we denote by $\mathcal{C}_{m}$ connecting a point on $\sigma_{1} \cup \sigma_{2}$ with the point $\left(\frac{1}{4}, 0\right)$, and

$$
m_{p}(p, a)>0, \quad(p, a) \in \Sigma^{+} .
$$

Throughout $\Sigma^{+}$, the level curves $\mathcal{C}_{L}$ and $\mathcal{C}_{m}$ intersect transversely, except along a unique curve which we denote by $\gamma$ connecting the points $\left(-\frac{7}{100}, \frac{4}{5 \sqrt{5}}\right)$ and $(1 / 4,0)$. Along $\gamma$,

$$
W(p, a) \equiv \frac{\Delta}{L}\left(L_{a} m_{p}-L_{p} m_{a}\right)
$$

vanishes. The curve $\gamma$ can be parametrized by $p, a=a^{\gamma}(p)$. Along it a is monotone decreasing with $p$. Above $\gamma, W<0$, and below $\gamma, W>0$. For $\widehat{m} \in\left(0, \frac{1}{\sqrt{5}}\right]$, the curves $\mathcal{C}_{\widehat{m}}$ and $\gamma$ do not intersect within the interior of $\Sigma^{+}$, and for $\widehat{m} \in\left(\frac{1}{\sqrt{5}}, 1\right)$, the curves $\mathcal{C}_{\widehat{m}}$ and $\gamma$ have a unique intersection within the interior of $\Sigma^{+}$. The points $(p, a) \in \gamma$ correspond to the unique folds or turning points of the bifurcation diagram, and the values assumed by $L=L(p, a)$ correspond to the values $L^{\gamma}(m)$ mentioned in Theorem 1.6. All intersections of the curve $\gamma$ with the curves $\mathcal{C}_{L}$ are transverse.

Proof. For a proof of Theorem 4.2, we refer the reader to Lemmas 3.2, 3.4, 3.5, 3.6, 3.7 and Theorems 3.8, 3.10 in [27], and to the references cited there.

In terms of the notation introduced in Section 2, the solutions corresponding to points $(p, a)$ lying above $\gamma$ are monotone spike solutions, and the solutions corresponding to points $(p, a)$ lying below $\gamma$ are monotone interface solutions. Actually, the lemma stated below and proven later in this section demonstrates that indeed $\gamma$ divides $\Sigma^{+}$into a spike region and an interface region, also for stationary solutions of arbitrary lap number.

Lemma 4.3. For any $k, k \in\{1,2, \ldots\}$, the locus of points in $\Sigma^{+}$which correspond to turning points for stationary solutions with lap number $k$ is given by the curve $\gamma$.

Similarly, it is easy to see that

Lemma 4.4. For any $k, k \in\{1,2, \ldots\}$, the locus of points in $\Sigma^{+}$which correspond to points of bifurcation of stationary solutions with lap number $k$ from the homogeneous solution is given by the curve $\sigma_{2}$.

The following alternative representation is also available.

Theorem 4.5. For $(p, a) \in \Sigma^{+}$setting $n(p, a)=\frac{1}{L} \int_{0}^{L} z^{2}(y) d y$, we may write

$$
L(p, a)=J_{0, p}, \quad m(p, a)=\frac{J_{1, p}}{J_{0, p}}, \quad n(p, a)=\frac{J_{2, p}}{J_{0, p}},
$$


where

$$
J_{i}(p, a)=\int_{\Gamma} z^{n} \sqrt{2}\left(\frac{z^{4}}{4}-\frac{z^{2}}{2}+a z+p\right) d z
$$

where $\Gamma$ denotes the piece of the curve

$$
\left(\frac{1}{2} v^{2} \equiv\right) \frac{1}{2} z_{y}^{2}=\frac{z^{4}}{4}-\frac{z^{2}}{2}+a z+p
$$

in the upper half of the $\left(z, z_{y}\right)$, connecting points which we denote by $\left(z_{0}(p, a), 0\right)$ and $\left(z_{1}(p, a), 0\right)$.

Moreover, $n_{a}=L_{a}=0$ along $\sigma_{0}, n=m^{2}$ along $\sigma_{1} \cup \sigma_{2}$, and $z_{0}(p, a)=z_{1}(p, a)$ along $\sigma_{2}$.

Proof. The proof of Theorem 4.5 relies on the information given in Lemma 3.4 and in the statement and proof of Proposition 3.1 of [27].

Lemma 4.6. For any $(p, a) \in \Sigma^{+}$, the energy of a monotone stationary solution corresponding to that $(p, a)$ is given by

$$
E(p, a)=\frac{J_{0}}{J_{0, p}}-a m(p, a)-p,
$$

or equivalently, by

$$
E(p, a)=-\frac{1}{3}(n(p, a)-p) .
$$

Proof. Using the fact that the first integral of a stationary solution may be written in the form (4.6), we note that in the notation of Theorem 4.2,

$$
\begin{gathered}
E=\frac{1}{L} \int_{0}^{L}\left\{\frac{1}{2} z^{4}-z^{2}+a z+p\right\} d x=\frac{1}{L} \int_{\Gamma}\left\{\frac{\frac{1}{2} z^{4}-z^{2}+a z+p}{v}\right\} d z \\
=\frac{1}{L}\left\{\frac{1}{2} J_{4, p}-J_{2, p}+a J_{1, p}+p J_{0, p}\right\} .
\end{gathered}
$$

From (4.6), it follows that $v v_{z}=z^{3}-z+a$; hence

$$
\frac{z^{4}}{v}=\frac{z^{2}}{v}-a \frac{z}{v}+z v_{z}
$$

Integrating the above expression over $\Gamma$ and integrating by parts, we obtain

$$
J_{4, p}=J_{2, p}-a J_{1, p}-J_{0} .
$$

Similarly, from (4.6) and (4.10), it follows that

$$
v=2 p \frac{1}{v}+\frac{3 a}{2} \frac{z}{v}-\frac{1}{2} \frac{z^{2}}{v}+\frac{1}{2} z v_{z} .
$$

Again, integrating the above expression over $\Gamma$ and integrating by parts, we obtain

$$
\frac{3}{2} J_{0}=2 p J_{0, p}+\frac{3 a}{2} J_{1, p}-\frac{1}{2} J_{2, p} .
$$

(This equation appears as equation (3.14) in [27].) Using (4.11) and (4.12) it is easy to see that (4.9) may be expressed as either (4.7) or (4.8).

In addition to the monotonicity lemmas of $[27,42,26]$, we also need

Lemma 4.7. $m_{a}(p, a)>0$ in $\Sigma^{+}$. 
Proof. Recall, as noted in Theorem 4.2, that $\gamma$ divides $\Sigma^{+}$into two regions, such that $W<0$ above $\gamma$ and $W>0$ below $\gamma$. Since $\Delta<0$ and $L_{p}, L_{a}$, and $m_{p}$ are positive throughout $\Sigma^{+}$(Theorem 4.2), it follows from the definition of $W(p, a)$ that, in the region $\{W>0\} \cap \Sigma^{+}, m_{a}>0$.

By continuity, it follows from (4.4) that, along $\mathcal{C}_{m}$ which cross $\gamma$, there exists a neighborhood in which $W<0$ and which lies in proximity to $\gamma$, in which $m_{a}>0$. If $m_{a}>0$ throughout the entire region in which $W<0$, we are done, so let us suppose that there exists a value of $\widehat{m}$ for which $m_{a}<0$. It would then follow, by (4.3), that the slope of the curve $\mathcal{C}_{\widehat{m}}$ must change sign (as a curve parametrized by $p$ ) within the region $W<0$. However, by (4.2), the slope of the curves $a_{p}^{L}(p)=-L_{p} / L_{a}$ remains negative for all curves $\mathcal{C}_{L}$ in this region. Thus, within the region $\{W<0\} \cap \Sigma^{+}$, there must be a point where a $\mathcal{C}_{L}$ curve and a $\mathcal{C}_{m}$ curve intersect nontransversely. However, at such a point necessarily $W(p, a)=0$ ( Theorem 4.2), yielding a contradiction.

Proof of Theorem 4.1. To prove (i), we first evaluate $E(p, a)$ along the boundaries of $\Sigma^{+}$. Note that by Theorems $4.2-4.5$, along $\sigma_{1}$

$$
n=m^{2}=\frac{1}{3}(1+\sqrt{1+12 p}),
$$

and along $\sigma_{2}$

$$
n=m^{2}=\frac{1}{3}(1-\sqrt{1+12 p}) .
$$

Therefore, we may conclude that along $\sigma_{1} \cup \sigma_{2}$

$$
p=\frac{3}{4} m^{4}-\frac{1}{2} m^{2} .
$$

Substituting this expression for $p$ into the second expression for the energy, and noting that $n=m^{2}$ along $\sigma_{1} \cup \sigma_{2}$ ( Theorem 4.5), we obtain that along $\sigma_{1}$

$$
E\left(p, a_{1}(p)\right)=-\frac{1}{2}\left(n-\frac{1}{2} n^{2}\right)=\frac{1}{4} m^{4}-\frac{1}{2} m^{2},
$$

and similarly, along $\sigma_{2}$,

$$
E\left(p, a_{2}(p)\right)=\frac{1}{4} m^{4}-\frac{1}{2} m^{2} .
$$

Since $\frac{1}{4} m^{4}-\frac{1}{2} m^{2}$ is clearly the energy of the trivial state $u(x) \equiv m$, (i) is proven.

In order to prove (ii), we examine the partial derivatives of $E(p, a)$. From the first expression for the energy and since $J_{0, p}=L$ (Theorem 4.5), it follows that

$$
E_{p}=\frac{-J_{0} J_{0, p p}}{J_{0, p}^{2}}-a m_{p}=-\frac{J_{0} L_{p}}{L^{2}}-a m_{p} .
$$

Since $z_{0}(a)=z_{1}(a)$ along $\sigma_{2}$ ( Theorem 4.5 ), it is easy to see that along $\sigma_{2}$

$$
J_{0}\left(a_{2}^{-1}(a), a\right)=\int_{\Gamma} v d z=0 .
$$

Hence integrating with respect to $p$,

$$
J_{0}(p, a)=\int_{a_{2}^{-1}(a)}^{p} J_{0, p} d p+J_{0}\left(a_{2}^{-1}(a), a\right),
$$


and noting that $J_{0, p}=L>0$, we obtain that $J_{0}>0$ throughout $\Sigma^{+}$. By (4.2)-(4.3) $L_{p}>0$ and $m_{p}>0$ throughout $\Sigma^{+}$; therefore it follows that

$$
E_{p}(p, a)<0 \quad(p, a) \in \Sigma^{+} .
$$

Similarly from the first and second expressions for the energy, we have that

$$
E_{a}(p, a)=\frac{-J_{0} J_{0, p a}}{J_{0, p}^{2}}-a m_{a}=\frac{-J_{0} L_{a}}{L^{2}}-a m_{a}
$$

and

$$
E_{a}(p, a)=-\frac{1}{3} n_{a}
$$

Since $m_{a}>0$ by Lemma 4.7 and since $L_{a}>0$ by (4.2), it follows from (4.17) that

$$
E_{a}(p, a)<0, \quad(p, a) \in \Sigma^{+} .
$$

Similarly from (4.18) and Theorem 4.5, it follows that

$$
E_{a}(p, a)=0 \text { along } \sigma_{0} .
$$

From the analyticity of $E(p, a)$ throughout $\Sigma^{+}$, the behavior of the derivatives of $E(p, a)$ in $\Sigma^{+}$, and from the information given above concerning the behavior of $E(p, a)$ along $\sigma_{1} \cup \sigma_{2}$ given in (4.14)-(4.15), it follows that in a neighborhood of the point $(1 / 4,0), E(p, a)$ uniformly approaches the value $-1 / 4$, independently of $m$.

In order to prove (iii) and (iv), it is useful to calculate the directional derivative of $E(p, a)$ along $\mathcal{C}_{m}$. Recalling that $m_{a}>0$ throughout $\Sigma^{+}$by Lemma 4.7, and $m_{p}>0$ by (4.3), it follows that the curves $\mathcal{C}_{m}$ may be parametrized as curves $a^{m}=a^{m}(p)$. It is now easy to calculate the directional derivative of $E(p, a)$ along $a^{m}(p)$ :

$$
\frac{d}{d p} E\left(p, a^{m}(p)\right)=E_{p}(p, a)-\frac{m_{p}}{m_{a}} E_{a}(p, a)=\frac{J_{0}}{\Delta L m_{a}} W(p, a) .
$$

Similarly, the directional derivative of $L(p, a)$ along $a^{m}(p)$ is given by

$$
\frac{d}{d p} L\left(p, a^{m}(p)\right)=L_{p}-\frac{m_{p}}{m_{a}} L_{a}=-\frac{L}{\Delta m_{a}} W(p, a) .
$$

We now recall that $m_{a}>0, J_{0}>0$, and $\Delta<0$ throughout the interior of $\Sigma^{+}$, and that within the interior of $\Sigma^{+}, W(p, a)=0$ vanishes only along the unique curve $\gamma$ where the turning points occur corresponding to the points where $L(p, a)=L^{\gamma}(m)$. Therefore, along curves $\mathcal{C}_{m}$, it follows from (4.20)-(4.21) that, if $L \neq L^{\gamma}(m)$, then $\frac{d E}{d L}=-J_{0} L^{-2}<0$, thus yielding the conclusions of claims (iii) and (iv) in the lemma.

In order to prove (v), it is necessary to consider what happens to $E(p, a)$ along the curves $\mathcal{C}_{L}$. In particular, we may calculate now that

$$
\frac{d}{d p} E\left(p, a^{L}(p)\right)=E_{p}(p, a)-\frac{L_{p}}{L_{a}} E_{a}(p, a)=-\frac{a L}{\Delta L_{a}} W(p, a) .
$$

As $a>0, L_{a}>0$, and $\Delta<0$ throughout $\Sigma^{+}$and since $W(p, a)$ vanishes only along the unique curve of turning points $\gamma$, we obtain that the curves $\mathcal{C}_{E}$ ( curves of constant energy ) and $\mathcal{C}_{L}$ intersect transversely everywhere in $\Sigma^{+}$except along the curve $\gamma$. Since, as noted in Theorem 4.2, the curves $\mathcal{C}_{L}$ always intersect the curve $\gamma$ transversely, and since the curves $\mathcal{C}_{E}$ and $\mathcal{C}_{L}$ do not intersect transversely along $\gamma$, we have that the curves $\mathcal{C}_{E}$ also always intersect the curve $\gamma$ transversely 
(to see this, just apply the definition of transversality). Thus we may conclude that $E$ cannot assume the same value twice along $\gamma$ and hence by looking at the values assumed by $E(p, a)$ at the endpoints of the curve $\gamma$, we may conclude that $E(p, a)$ is monotone decreasing along the curve $\gamma$. Furthermore, it follows from (4.22) and Lemma 4.7 that the curves $\mathcal{C}_{E}$ may be parametrized as $a^{E}(p)$ and from $(4.22)$ it follows that, along the curves $\mathcal{C}_{E}, E\left(p, a^{E}(p)\right)$ is monotone decreasing when $W<0$ and $E\left(p, a^{E}(p)\right)$ is monotone increasing when $W>0$.

With this information, we show now that $E(L, i) \neq E(L, s)$ if $L \neq L^{\gamma}$. The proof is by contradiction. Suppose that for some $\bar{L}$ and $\bar{m}$ such that $\bar{L} \neq L^{\gamma}(\bar{m})$, $E(\bar{L}(\bar{m}), i)=E(\bar{L}(\bar{m}), s)$, and let us consider the region of $\Sigma^{+}$bounded by the curve $\mathcal{C}_{\bar{m}}, L \in\left(L^{\gamma}(\bar{m}), \bar{L}\right)$ (both in the region in which $W>0$ and in the region in which $W<0$ ), and by the curve $\mathcal{C}_{\bar{L}}$ which intersects the curve $\mathcal{C}_{\bar{m}}$ at the points where $L=\bar{L}$. Note that this region is intersected by the curve $\gamma$. Let us consider the part of this region which lies above the curve $\gamma$ and the values assumed by $E(p, a)$ along the boundary of this region. Noting now that $E(p, a)$ is decreasing along $\gamma$ and decreasing along $\mathcal{C}_{\bar{L}}$ and $\mathcal{C}_{\widehat{m}}$ when $W<0$, we conclude that there must exist a point on $\mathcal{C}_{\bar{m}}$ which lies above $\gamma$ where $E(p, a)=E^{0}$, where $E^{0}$ denotes the value of the energy assumed at the point where $\mathcal{C}_{\bar{L}}$ intersects $\gamma$. Since, by (4.16), (4.19), and the boundary data (4.14)-(4.15), there is a unique curve $a^{E}=a^{E}(p)$ along which

$$
E\left(p, a^{E}(p)\right)=E^{0} .
$$

This implies that there must be a curve lying above $\gamma$ along which $E=E^{0}$. Let us also look now at the values assumed by the energy function $E(p, a)$ at the intersection points of the curves $\mathcal{C}_{\bar{L}}$ and $\mathcal{C}_{\bar{m}}$. At these intersection points,

$$
E(p, a)=E(\bar{L}, i)=E(\bar{L}, s) \equiv \bar{E} .
$$

By the construction and by the monotonicity of $E(p, a)$ along $\mathcal{C}_{\bar{m}}$ it follows that $\bar{E}<E^{0}$. However, let us consider the curve $\mathcal{C}_{\bar{E}}$ along which $E=\bar{E}$. Clearly, by the monotonicity of $E$ along the curves $\mathcal{C}_{\bar{m}}$ and $\mathcal{C}_{\bar{L}}$, this curve cannot exit the region defined above except through the points of intersection of the two curves $\mathcal{C}_{\bar{L}}$ and $\mathcal{C}_{\bar{m}}$. Therefore, the curve $\mathcal{C}_{\bar{E}}$ must intersect the curve $\mathcal{C}_{E^{0}}$ which we described above. However, as we have noted, $E^{0} \neq \bar{E}$, and hence a contradiction is obtained.

From the above lemma, the next theorem follows essentially as a corollary.

Theorem 4.8. For any $L>0$ and any $m \in[0,1]$, let $k$ be the number of interface solutions. Then,

$$
E\left(M_{i}^{1 \pm}\right)<E\left(M_{i}^{2 \pm}\right)<\cdots<E\left(M_{i}^{k \pm}\right)
$$

where $E\left(M_{i}^{j \pm}\right)=E\left(M_{i}^{j \pm}(L, m)\right)$ denotes the energy of an interface solution with lap number $j$.

Proof. For any $L>0$ and any $m \in[0,1]$, it suffices to consider two branches of interface solutions, $M_{i}^{i \pm}(L, m), M_{i}^{(i+1) \pm}(L, m), \quad 1 \leq i \leq k-1$. By Lemma 2.1,

$$
M_{i}^{j \pm}(L, m)=M_{i}^{1 \pm}\left(\frac{L}{j}, m\right)
$$

and

$$
M_{i}^{(j+1) \pm}(L, m)=M_{i}^{1 \pm}\left(\frac{L}{j+1}, m\right) .
$$


Hence,

$$
M_{i}^{j \pm}(L, m)=M_{i}^{(j+1) \pm}\left(\frac{j+1}{j} L, m\right),
$$

and therefore, by Theorem 4.1,

$$
E\left(M_{i}^{j \pm}(L, m)\right)<E\left(M_{i}^{(j+1) \pm}(L, m)\right) .
$$

For the unstable region in which there are no spike solutions, using Theorem 4.8 it is easy to obtain a complete ordering of all stationary solutions:

Corollary 4.9. For any $L>0$ and any $m \in\left[0, \frac{1}{\sqrt{5}}\right]$, let $k$ be the number of interface solutions. Then

$$
E\left(M_{i}^{1 \pm}\right)<E\left(M_{i}^{2 \pm}\right)<\cdots<E\left(M_{i}^{k \pm}\right)<E^{h} .
$$

Proof. By the statement of Theorem 4.8, it only remains to verify the last inequality. Noting that by Lemma 2.1

$$
M_{i}^{k \pm}(L, m)=M_{i}^{1 \pm}\left(\frac{L}{k}, m\right)
$$

and by definition of $k, L / k \geq L_{\min }(m)$, it follows from Theorem 4.1 parts (i) and (iii) that $E\left(M_{i}^{k \pm}\right)<E^{h}$.

For the transitional and metastable regions, we have the following partial results.

Theorem 4.10. For any $L>0$ and any $m \in\left(\frac{1}{\sqrt{5}}, 1\right)$, let $k$ be the number of interface solutions and let $k_{s}$ be the number of spike solutions. Then for $L$ sufficiently large, there exists a $j \leq k$ such that

$$
E\left(M_{i}^{1 \pm}\right)<\cdots<E\left(M_{i}^{j \pm}\right)<E^{h}<E\left(M_{s}^{1 \pm}\right)<\cdots<E\left(M_{s}^{k_{s} \pm}\right) .
$$

Proof. Recall that in the transitional region $(2.2) k_{s} \leq k$, while as discussed in $\S 2$ in the metastable region, $k_{s}=k$.

Note first that repeating the steps of the proof of Theorem 4.8 for spike solutions, for any $1 \leq j \leq k_{s}-1$

$$
E\left(M_{s}^{(j+1) \pm}\right)>E\left(M_{s}^{j \pm}\right)
$$

and from parts (i) and (iv) of Theorem 4.1, it follows that, for any $1 \leq j \leq k_{s}$,

$$
E^{h}<E\left(M_{s}^{j \pm}\right) .
$$

Furthermore, from parts (ii) and (iii) of Lemma 4.1 for $L$ sufficiently large,

$$
E\left(M_{i}^{j \pm}\right)<E^{h},
$$

for some $j \in\{1, \ldots, k\}$. Combining inequalities (4.25)-(4.27) yields the ordering prescribed by the theorem.

Remark. Our results concerning the energy levels of the stationary solutions encompass all the energy results given by Carr, Gurtin and Slemrod in Proposition 8.1, and Theorems 8.2 and 8.3 of [13] when $W(u)$, the free energy per unit volume, is a quartic polynomial. The expressions for the first two terms given in Theorem 8.1 of [13] for the energy of the "Maxwell solution" follow immediately here from (4.20) and (4.21). 
We conclude this section with a proof of Lemma 4.3 which now follows easily from information used in proving energy separation.

Proof. That the claim of the lemma is true for $k=1$ follows from Theorem 4.2. For $k>1$, we note that, by the scaling arguments used in the proof of Theorem 4.8 ,

$$
m_{k}(p, a)=m(p, a) \text { and } L_{k}(p, a)=k L(p, a),
$$

where $m_{k}(p, a)$ and $L_{k}(p, a)$ are respectively the mean mass and period of the stationary solution with lap number $k$ corresponding to the point $(p, a) \in \Sigma^{+}$. Following the arguments in Lemma 3.5 of [27], the locus of turning points is determined by the condition that there exists a $\lambda_{k} \neq 0$ such that

$$
\nabla L_{k}=\lambda_{k} \nabla m_{k}
$$

which by (4.28) is equivalent to the existence of a $\lambda \neq 0$ such that

$$
\nabla L=\lambda \nabla m .
$$

By Lemma 3.5 of [27], this later condition yields precisely the locus of points $(p, a) \in$ $\Sigma^{+}$corresponding to the turning points for monotone stationary solutions.

\section{Hyperbolicity OF EQUiLIBRIA}

In this section we prove a nondegeneracy theorem, Theorem 5.1 below. It both is of independent interest and also serves as a tool in the discussions of stability in the next section. In what follows we denote by primes derivatives with respect to $x$.

Theorem 5.1. Away from points of bifurcation from the homogeneous solution and from turning points, zero is not in the spectrum of the linearization around a stationary solution.

Proof. First of all note that by Lemma 1.2, it suffices to prove nondegeneracy for the nonlocal reaction-diffusion equation only. Thus suppose $u(x)$ is a solution of the nonlocal reaction-diffusion equation for some value of $L$ and $m$; i.e.,

$$
u^{\prime \prime}+f(u)-\frac{1}{L} \int_{0}^{L} f(u) d x=0, \quad x \in(0, L), \quad u^{\prime}(0)=u^{\prime}(L)=0,
$$

where $f(u)=u^{3}-u$. The associated eigenvalue problem is

$$
\mathcal{L} \phi=\lambda \phi,
$$

where

$$
\mathcal{L} \phi=\phi^{\prime \prime}+f^{\prime}(u) \phi-\frac{1}{L} \int_{0}^{L} f^{\prime}(u) \phi d x
$$

with Neumann boundary conditions, acting on functions $\phi \in \dot{L^{2}}([0, L])$. It is proven via the following two lemmas that away from points of bifurcation from a homogeneous solution and away from turning points, zero is not in the spectrum of (5.2). This then completes the proof of Theorem 5.1.

The following lemma extends the results of Proposition 4.1.3 in Schaaf [47] to the case of Neumann boundary conditions. 
Lemma 5.2. If $u(x)$ is not the homogeneous solution $u(x) \equiv m$, then the associated local eigenvalue problem

$$
\psi^{\prime \prime}-f^{\prime}(u) \psi=\mu \psi, \psi^{\prime}(0)=\psi^{\prime}(L)=0
$$

has no zero eigenvalues.

Proof. Note that if $u(x)$ solves (5.1), then it also solves the local problem

$$
u^{\prime \prime}-f(u)+a=0, x \in(0, L), \quad u^{\prime}(0)=u^{\prime}(L)=0 .
$$

This means in particular that it is the solution of the initial value problem

$$
u^{\prime \prime}=u^{3}-u+a, \quad u^{\prime}(0)=0, u(0)=q,
$$

where $q \equiv q(L, m)$, or alternatively $q \equiv q(L, p)$ (see $[27]$ ).

Differentiating with respect to $q$ (keeping $a$ fixed), we get the following initial value problem for $u_{q}=w$ :

$$
w^{\prime \prime}=\left(3 u^{2}-1\right) w, \quad w^{\prime}(0)=0, w(0)=1,
$$

Also, $u^{\prime}(L)=0$. Clearly, if $q$ is given, $L$ is a function of $a$ and $q$, or alternatively of $a$ and $p$ as $q_{p}>0$ throughout $\Sigma^{+}([42])$. Therefore, differentiating $u^{\prime}(L)$ with respect to $q$,

$$
u^{\prime \prime} \frac{\partial L}{\partial q}+w^{\prime}=0
$$

at $x=L$. On the other hand, at $x=L$,

$$
u^{\prime \prime}=g(q)^{3}-g(q)+a,
$$

where either $g(q)=q$ or else it is the other point of intersection of the level curve $H\left(u, u^{\prime}\right)=p$ of the Hamiltonian

$$
H\left(u, u^{\prime}\right)=\frac{1}{2}\left(u^{\prime}\right)^{2}-\frac{1}{4} u^{4}+\frac{1}{2} u^{2}-a u
$$

with the $u^{\prime}=0$ axis.

Thus, explicitly indicating the dependence of $q$ and $L$ on $a$ and $p$, we have

$$
w^{\prime}(L(a, p), q(a, p))=-\frac{\partial L}{\partial q}\left(g^{3}(q)-g(q)+a\right) .
$$

Now suppose that $\psi(x)$ is a zero-eigenfunction of (5.3). Then, normalizing, we see that $\psi$ satisfies the same initial value problem (5.6) as $w$, so by uniqueness of the solution of the initial value problem and from the second boundary condition in (5.3),

$$
0=\psi^{\prime}(L)=-\frac{\partial L}{\partial q}\left(g^{3}(q)-g(q)+a\right)
$$

Differentiating

$$
p+\frac{1}{4} q^{4}-\frac{1}{2} q^{2}+a q=0
$$

with respect to $p$, it follows that if $\psi$ is a zero-eigenfunction, we must have

$$
0=\frac{\partial L}{\partial p} \frac{d g(q)}{d q}\left(g^{3}(q)-g(q)+a\right)^{2}
$$

However, from [42] we know that $L$ is monotone in $p, d g / d q$ is nonzero by inspection of the phase plane ( or see [42] ), and $g(q)^{3}-g(q)+a=0$ only for the homogeneous 
solution. Thus, if $u(x)$ is not the homogeneous solution, (5.3) does not admit a zero-eigenfunction.

Note that though the following lemma is stated for $f(u)=-u+u^{3}$, it does not depend on the particular form of $f(u)$.

Lemma 5.3. Let $u$ be any stationary solution of the nonlocal problem. If $u$ is not a turning point solution and if the local problem (5.3) is strongly nondegenerate at $u$, then so is the nonlocal problem.

Proof. Suppose we are not at a turning point and suppose that the nonlocal problem is not strongly nondegenerate; then there exists a zero eigenfunction, i.e. a function $w$ such that

$$
\begin{gathered}
w^{\prime \prime}+\left(-3 u^{2}+1\right) w-\frac{1}{L} \int_{0}^{L}\left(-3 u^{2}+1\right) w d x=0, \quad x \in(0, L), \\
w^{\prime}(0)=w^{\prime}(L)=0, \text { and } \frac{1}{L} \int_{0}^{L} w d x=0 .
\end{gathered}
$$

On the other hand, away from turning points, the derivative of $u$ with respect to $m, u_{m}$, is well-defined and satisfies

$$
\begin{gathered}
u_{m}{ }^{\prime \prime}+\left(-3 u^{2}+1\right) u_{m}-\frac{1}{L} \int_{0}^{L}\left(-3 u^{2}+1\right) u_{m} d x=0, \quad x \in(0, L), \\
u_{m}{ }^{\prime}(0)=u_{m}{ }^{\prime}(L)=0, \text { and } \frac{1}{L} \int_{0}^{L} u_{m} d x=1 .
\end{gathered}
$$

Multiplying (5.7) by $u_{m}$ and (5.8) by $w$, integrating, and subtracting, we get that

$$
\int_{0}^{L}\left(-3 u^{2}+1\right) w d x=0
$$

but then $w$ is also an eigenfunction corresponding to the zero eigenvalue of the local problem.

This theorem allows us to conclude that, away from points of bifurcation from the homogeneous solution ( which by [42] are known to be the only points of bifurcation which occur ) and from turning points, the Conley indices of all stationary solutions are pointed spheres of some dimension.

\section{Stability}

We shall denote by $W^{u}$ the (local) unstable manifold of a solution. The main result of this section is

Theorem 6.1. $\operatorname{dim} W^{u}\left(M_{i}^{k \pm}\right)=k-1 ; \operatorname{dim} W^{u}\left(M_{s}^{k \pm}\right)=k$.

Proof. First we deal with $M_{i}^{k \pm}$ solutions in the unstable case.

Lemma 6.2. In the unstable case $\operatorname{dim}\left(M_{i}^{k \pm}\right)=k-1$. 
Proof. Consider the situation for $L$ slightly above the $k$-th bifurcation point. It is easily checked that (a) the homogeneous solution $M_{h}$ has index $\Sigma^{k}$; (b) the solutions $M_{i}^{k \pm}$ have indices $\Sigma^{d}$ for some $d$ by Theorem 5.1 ; (c) the set $S=$ $M_{h} \cup M_{i}^{k \pm} \cup C\left(M_{h}, M_{i}^{k \pm}\right)$ is an isolated invariant set with index $\Sigma^{k-1}$ (by continuation with respect to $L$ to the trivial solution to a value of $L$ slightly below the bifurcation point); therefore the homology mod 2 complex of its index, $H_{*}(h(S))$ is $\left(0, \ldots, \mathbf{Z}_{2}, 0, \ldots\right)$ where the non-zero entry is located in the $(k-1)^{t h}$ place and hence it is one-dimensional, and (d) $\left\{M_{i}^{k+}, M_{i}^{k-}, M_{h}\right\}$ is a Morse decomposition for that set (this follows from energy considerations). We have that the connection matrix for that Morse decomposition is

$$
\tilde{\Delta}=\left[\begin{array}{lll}
0 & 0 & \alpha \\
0 & 0 & \alpha \\
0 & 0 & 0
\end{array}\right] .
$$

The conclusion that $\tilde{\Delta}(1,2)=0$ comes from energy considerations. By the $\mathbf{Z}_{2}$ symmetry of the equation, $C\left(M_{h}, M_{i}^{k+}\right)$ has the same structure as $C\left(M_{h}, M_{i}^{k-}\right)$, and hence since we are working over $\mathbf{Z}_{2}$, the entries $\tilde{\Delta}(1,3)$ and $\tilde{\Delta}(2,3)$ must be identical. Now we invoke the rank condition [23], which in this case states that

$$
\operatorname{dim} H_{*}(h(S))=\operatorname{dim} \operatorname{ker}(\tilde{\Delta})-\operatorname{rank}(\tilde{\Delta}) .
$$

Since

$$
\operatorname{dim} \operatorname{ker}(\tilde{\Delta})+\operatorname{rank}(\tilde{\Delta})=3
$$

and (c) above means $\operatorname{dim} H_{*}(h(S))=1$, we have that the rank of $\tilde{\Delta}$ is 1 , so that $\alpha$ is an isomorphism. But that means that the $k-1$-st singular homology group of $h\left(M_{i}^{k \pm}\right)$ is $\mathbf{Z}_{2}$, which means by the nondegeneracy result of Theorem 5.1 and the rank condition that $d=k-1$. That the index does not change as we increase $L$ also follows from Theorem 5.1.

Lemma 6.3. In both the metastable and transitional cases, $\operatorname{dim}\left(M_{i}^{k \pm}\right)=k-1$.

Proof. We use a continuation argument. By Theorem 5.1, away from turning points and points of bifurcation from the homogeneous solution, all equilibria are hyperbolic. In the notation of Theorem 4.2, all turning points lie along a curve $\gamma$, while all the points of bifurcation from the homogeneous solution lie along the curve $\sigma_{2}$. The curve $\gamma$ admits a parametrization $a=a^{\gamma}(p)$, while $\sigma_{2}$ can be parametrized as $a=a_{2}(p)$ (see Lemma 1.7). Along both curves $a$ is monotone decreasing as a function of $p$.

Let $\Sigma_{i}^{+}$denote the region enclosed by the curves $\gamma_{1}, \sigma_{2}$ and $\sigma_{0}$. By the arguments of $[42,27]$ points $(p, a)$ in $\Sigma_{i}^{+}$are in one to one correspondence to the monotone interface solutions $\left(M_{i}^{1 \pm}\right)$. Furthermore, it is not hard to show that the curves $\mathcal{C}_{m}$ with $m=1 / \sqrt{5}$ and $m=1 / \sqrt{3}$ subdivide $\Sigma_{i}^{+}$into three sets $\Sigma_{i}^{u+}, \Sigma_{i}^{t+}, \Sigma_{i}^{m+}$, which correspond to interface solutions in unstable, transitional, and metastable regions, respectively.

We proceed to prove the lemma for $M_{i}^{1 \pm}$ only, since it is easy to see that these three regions are invariant under the scalings of Lemma 2.1, since $\gamma$ is thus invariant by Lemma 4.3. Fix a point $\left(p^{*}, a^{*}\right)$ in $\Sigma_{i}^{m+}$. From the geometry of the curves $\mathcal{C}_{m}$ it is clear that by following any path $\Gamma$ along which $a$ is non-increasing and $p$ is decreasing, we shall sooner or later hit a point $\left(p^{* *}, a^{* *}\right)$ in $\Sigma_{i}^{u+}$. Along $\Gamma$ the 
solution $M_{i}^{1 \pm}$ remains hyperbolic by Lemma 5.1, since we do not intersect either $\gamma$ or $\sigma_{2}$. Therefore the dimension of the unstable manifold is conserved along $\Gamma$.

Lemma 6.4. In both the metastable and transitional cases $\operatorname{dim}\left(M_{s}^{k \pm}\right)=k$.

Proof. The proof is similar to that of Lemma 6.2, except that we consider the situation slightly above the $k$-th turning point $L^{k}$. Then the set $M_{i}^{k \pm} \cup M_{s}^{k \pm} \cup$ $C\left(M_{s}^{k \pm}, M_{i}^{k \pm}\right)$ is an isolated invariant set with index $\overline{0}$ and $\left\{M_{i}^{k+}, M_{i}^{k-}, M_{s}^{k+}\right.$, $\left.M_{s}^{k-}\right\}$ is a Morse decomposition with connection matrix

$$
\tilde{\Delta}=\left[\begin{array}{llll}
0 & 0 & \beta & \gamma \\
0 & 0 & \gamma & \beta \\
0 & 0 & 0 & 0 \\
0 & 0 & 0 & 0
\end{array}\right],
$$

where we have again used symmetry and energy considerations. Since the rank of this matrix is 2 , we conclude that either $\beta$ or $\gamma$ is an isomorphism. It is not hard to see that this implies that $h\left(M_{s}^{k \pm}\right)=\Sigma^{k}$. Again, increasing $L$ does not change the index by Theorem 5.1 .

Remark. Note that by considering the invariant set $M_{i}^{k+} \cup M_{s}^{k+} \cup C\left(M_{i}^{k+}, M_{i}^{k+}\right)$ and the above argument, we can prove, for $L$ sufficiently close to a fold point, that $\beta$ is an isomorphism (and consequently, $\gamma$ is the zero homomorphism). This means that close to the $k$-th turning point there exists a heteroclinic connection between $M_{s}^{k+}$ and $M_{i}^{k+}$, as well as between $M_{s}^{k-}$ and $M_{i}^{k-}$. This fact will be used later on.

Remark. Alternatively, the dimensions of the unstable manifolds can be computed using the nondegeneracy result of the previous section. Note first the dimension

of the unstable manifold for large $L$ (small $\epsilon$ ) for $M_{i}^{k \pm}$ was computed in [9], and more recently by different methods in [44]. For the case of spike solutions in the transitional case, the dimension of the unstable manifold can be easily obtained by a local bifurcation analysis at the point of coalescence of the turning point with bifurcation from the homogeneous solution (see [43] and [16]). Now using the nondegeneracy results of the previous section and continuity arguments as in the proof of Lemma 6.3 above, the desired results are obtained.

Remark. Note that while it is possible to homotopy the nonlocal problem to an associated local problem and to show in this way that the index of instability is identical for the nonlocal and associated local problems [24], these methods do not allow us to determine the number of zero eigenvalues.

\section{Structure of the ATtractor}

In this section we prove existence of certain kinds of heteroclinic connections between stationary solutions. We concentrate primarily on the harder metastable case. The main tools are the energy separation results obtained above and the Conley connection matrix [14],[23]; the reader is referred to those papers for the relevant background. First, we remark on the unstable case. Here the best results are due to Mischaikow [35]: 
7.1. The unstable case. If $L \in\left(k \pi / \sqrt{1-3 m^{2}},(k+1) \pi / \sqrt{1-3 m^{2}}\right)$, a Morse decomposition under the flow-induced order for the attractor is given by $\left\{M_{i}^{1 \pm}, \ldots\right.$, $\left.M_{i}^{k \pm}, M_{h}\right\}$. In this case it is possible to compare the global attractor of the viscous Cahn-Hilliard homotopy with that of the Chaffee-Infante ( Allen-Cahn [2] ) equation defined on the interval $[0, \pi]$ :

$$
\begin{gathered}
u_{t}=u_{x x}+\lambda^{2}\left(u-u^{3}\right), \\
u(0, t)=u(\pi, t)=0 .
\end{gathered}
$$

For any $\lambda>0$, for the Chaffee-Infante ( Allen-Cahn ) equation there is a trivial stationary solution which we denote as $M_{h}$ in addition to $2 k(=2[\lambda])$ nontrivial stationary states which come in pairs having lap number $l(j), j \in\{1,2, \ldots, k\}$, which we denote as $M^{j \pm}$.

The precise relation between the two attractors is as follows:

Theorem 7.1. If $g$ is a gradient system with attractor $A_{g}^{\lambda}$ having the bifurcation diagram of the Chaffee-Infante equation with bifurcation parameter $\lambda$, then there exists a flow $\psi^{\lambda}$ on a ball $D^{[\lambda]}$, such that the flow on the attractor $A_{g}^{\lambda}$ is semiconjugate to the flow $\psi^{\lambda}$.

This follows from Theorem 1.2 of [35]. The proof in [35] proceeds by verifying four assumptions A1-A4 which we now review briefly.

- A1 is an assumption on the existence of a compact attractor. For the entire Cahn-Hilliard homotopy, this is proved in Theorem 3.4.

- A2 says that the sets $\left\{M_{i}^{1 \pm}, M_{i}^{2 \pm}, \ldots, M_{i}^{j \pm}, M_{h}\right\}$ for $j \in\{1,2, \ldots, k\}$ form a Morse decomposition for the attractor; this follows from the energy considerations of Theorem 4.1.

- A3 is a statement concerning the cohomology Conley indices of the Morse sets; this follows from the instability index computations of Lemma 6.2.

- A4 is a statement concerning the structure of the connection matrix for this Morse decomposition. More precisely, using Alexander-Spanier cohomology with coefficients in $\mathbf{Z}$, it is shown in [35] that the connection matrix is the $2[\lambda] \times 2[\lambda]$ matrix $\Delta$ with the structure

$$
\Delta=\left[\begin{array}{ccc}
O_{2 \times 2} & O_{2 \times 2} & \cdots \\
C & O_{2 \times 2} & \cdots \\
\cdots & C & \cdots
\end{array}\right],
$$

where the subdiagonal $2 \times 2$ blocks $C$ have the form

$$
C=\left[\begin{array}{ll}
1 & -1 \\
1 & -1
\end{array}\right]
$$

It is easy to see that if instead we use singular homology with coefficients in $\mathbf{Z}_{2}$ the resulting connection matrix will be

$$
\Delta=\left[\begin{array}{cccc}
O_{2 \times 2} & D & \cdots & \cdots \\
\cdots & O_{2 \times 2} & D & \cdots \\
\cdots & \cdots & \cdots & \cdots \\
\cdots & \cdots & \cdots & O_{2 \times 2}
\end{array}\right]
$$


where the $D$ blocks have the form

$$
D=\left[\begin{array}{ll}
1 & 1 \\
1 & 1
\end{array}\right]
$$

The fact that the connection matrix in this case has the requisite structure follows from the bifurcation analysis of [27]; see [35] for details of the argument.

In other words, the attractor for the viscous Cahn-Hilliard homotopy contains all the connections of the Chaffee-Infante attractor.

Corollary 7.2. In the unstable region, for all $\alpha \in[0,1]$ there exist heteroclinic connections from every $M_{i}^{k \pm}$ solution to every $M_{i}^{j \pm}$ solution, $1 \leq j<k \leq n$. Furthermore, $M_{h}$ is connected to every $M_{i}^{k \pm}$ solution, $1 \leq k \leq n$.

In the metastable case Mischaikow's theorem cannot be applied, as there is a much richer set of equilibria and clearly, for example, assumption A2 does not hold.

7.2. The metastable case. For reasons described in $\S 8$, we cannot at this stage obtain exhaustive information about the structure of the attractor even for $\alpha$ close to 1 . However, Theorem 4.10 allows us to say something about certain "slices" of the attractor in this case.

Fix $m \in(1 / \sqrt{3}, 1)$, so that we are in the metastable case. Let $\tilde{L}_{k}<L<\tilde{L}_{k+1}$, where $\tilde{L}_{k}$ is the $k$-th turning point, $k \geq 2$ for simplicity. There is a maximal integer $0<r_{m} \equiv r_{m}(L) \leq k$, such that for $k \leq r_{m}$ we have

$$
E\left(M_{i}^{1 \pm}\right)<\cdots<E\left(M_{i}^{r_{m} \pm}\right)<E\left(M_{h}\right) .
$$

It is clear that $r_{m}(L)$ is an increasing function of $L$.

We present now two auxiliary lemmas which will aid in understanding the structure of the set $\left\{M_{i}^{j \pm}, j=1, \ldots, r_{m}(L)\right\}$. For any $m \in(0,1)$, let

$$
E_{r e l} \equiv E(p, a)-\frac{1}{4} m^{4}+\frac{1}{2} m^{2}
$$

Noting that $E_{r e l}=E(p, a)-E_{h}$, clearly $E_{r e l}<0$ for all interface solutions in the unstable region as well as for the set $\left\{M_{i}^{j \pm}, j=1, \ldots, r_{m}(L)\right\}$. Let us denote by $\Sigma_{E_{r e l}}^{+}$the subset of $\Sigma^{+}$on which $E_{r e l}<0$. With regard to $\Sigma_{E_{r e l}}^{+}<0$, we have the following description:

Lemma 7.3. There exists a unique monotone decreasing curve $\gamma_{r e l}$, connecting the points $\left(-\frac{7}{100}, \frac{4}{5 \sqrt{5}}\right)$ and $\left(\frac{1}{4}, 0\right)$, which lies below $\gamma$ such that $\Sigma_{E_{\text {rel }}}^{+}$is bounded by the curves $\sigma_{0}, \sigma_{2}$, and $\gamma_{\text {rel }}$.

Proof. It follows from Theorem 4.1 that $E_{r e l}=0$ along $\sigma_{1} \cup \sigma_{2}$ and by (4.16), $E_{p}<0$; hence $E_{r e l}<0$ along $\sigma_{0}$. From Theorem 4.1 we obtain looking along curves $\mathcal{C}_{m}$ that

$$
\frac{d}{d p} E_{r e l}\left(p, a^{m}(p)\right)>0 \text { above } \gamma, \quad \frac{d}{d p} E_{r e l}\left(p, a^{m}(p)\right)<0 \text { below } \gamma ;
$$

hence, in particular, we may conclude that $E_{r e l}>0$ along $\gamma$, the locus of turning points.

We claim similarly that

$$
\frac{d}{d p} E_{r e l}\left(p, a^{L}(p)\right)>0 \text { above } \gamma, \quad \frac{d}{d p} E_{r e l}\left(p, a^{L}(p)\right)<0 \text { below } \gamma .
$$


This can be demonstrated as follows:

It is easy to calculate that

$$
\begin{aligned}
& \frac{d}{d p} E_{r e l}\left(p, a^{L}(p)\right) \\
& \quad=\left[E_{p}-\frac{m_{p}}{m_{a}} E_{a}\right]+\left[\frac{m_{p}}{m_{a}}-\frac{L_{p}}{L_{a}}\right] E_{a}+\left[m_{p}-m_{a} \frac{L_{a}}{L_{p}}\right]\left(1-m^{2}\right) m,
\end{aligned}
$$

which using (4.20) and (4.21) becomes

$$
=\frac{W}{\triangle}\left[\frac{J_{0}}{L m_{a}}+\frac{E_{a} L}{m_{a} L_{a}}+\frac{L}{L_{a}}\left(1-m^{2}\right) m\right] .
$$

Employing (4.17), this further simplifies to yield

$$
\frac{d}{d p} E_{r e l}\left(p, a^{m}(p)\right)=\frac{W L}{\triangle L_{a}}\left[m-m^{3}-a\right] .
$$

To ascertain the sign of $\frac{d}{d p} E_{r e l}\left(p, a^{L}(p)\right)$, we set

$$
F=m-m^{3}-a,
$$

and note that (see [42]) $F=0$ along $\sigma_{1} \cup \sigma_{2}$ and $\frac{d}{d p} F\left(p, a^{m}(p)\right)=-\frac{d}{d p} a^{m}(p)>0$. Hence, $F>0$ throughout the interior of $\Sigma^{+}$. Since

$$
L_{p}, L_{a}, m_{p}, m_{a}>0
$$

by Theorem 4.2 and Lemma 4.7, and since $W<0$ above $\gamma$ and $W>0$ below $\gamma$, (7.2) is shown to hold.

We may now conclude that there exists a unique curve $\gamma_{\text {rel }}$ which lies below $\gamma$. From (7.1), (7.2) and (7.3), it follows now that $\gamma_{r e l}$ may be parametrized as a monotone decreasing curve $a^{E_{r e l}}=a^{E_{r e l}}(p)$ as described in the lemma.

Let us define $I_{k}=\left\{(a, p) \in \Sigma^{+} \mid 2 k\right.$ is $\left.\begin{array}{c}\text { the maximal number of interface solutions with } \\ \text { energy less than } E_{h} \text { in an interval of length } L(a, p)\end{array}\right\}$. We then have the following lemma giving information on the shape of the domains $I_{k}$.

Lemma 7.4. The domain $\Sigma_{E_{\text {rel }}}^{+}$can be partitioned into $I_{k}, k=1,2, \ldots$, connected regions, such that any point $(a, p) \in \Sigma_{E_{r e l}}^{+} \cap I_{k}$ may be connected to a point $(0, p) \in$ $\Sigma_{E_{\text {rel }}}^{+} \cap I_{k}$ by a continuous curve lying entirely within $I_{k}$.

Proof. Let $m \in(0,1)$ be fixed and denote $\tilde{\mathcal{C}}_{m}=\mathcal{C}_{m} \cap \Sigma_{E_{r e l}}^{+}$. By Lemma 7.3, $\gamma_{\text {rel }}$ lies below $\gamma$. Hence it follows from Theorem 4.2 that, along $\tilde{\mathcal{C}}_{m}, L(a, p)$ increases from a minimum value $L_{\text {min }}(m)$ attained at the intersection of $\tilde{\mathcal{C}}_{m}$ with $\sigma_{2} \cup \gamma_{\text {rel }}$ towards infinity as the point $\left(0, \frac{1}{4}\right)$ is approached. Since by its definition $E_{r e l}$ denotes the specific relative free energy of a solution, $E_{\text {rel }}$ of a non-monotone interface solution is $E_{\text {rel }}$ of any (identical) maximal monotone segments of which it is composed. Thus when $L(a, p)>2 L_{\min }(m)$, there exist at least 4 interface solutions with energy less than $E_{h}$. In this manner, we define

$$
k(a, p) \equiv\left[\frac{L(a, p)}{L_{\min }(m)}\right]
$$


(where square brackets denote integer part of a number), which increases from 1 to $\infty$ as $L$ increases along $\tilde{\mathcal{C}}_{m}$ from $L_{\text {min }}(m)$ to $\infty$. The locus of points $(a, p) \in \Sigma_{E_{r e l}}^{+}$ where $k(a, p)=1,2,3, \ldots$ constitute unique curves which may be denoted as $\mathcal{C}_{k}$ which connect the point along $\sigma_{0}$ at which $L(a, p)=2 \pi k$ with the point $\left(0, \frac{1}{4}\right)$. Clearly the curves $\mathcal{C}_{k}$ demarcate the boundaries of the regions $I_{k}, k=1,2,3, \ldots$. See Figure 2.

Now fix $m \in\left(\frac{1}{\sqrt{3}}, 1\right)$ so that we are in the metastable region again, and let $\mathcal{A}_{\alpha}^{L, m}$ denote the attractor where the $L$ dependence as well as the $\alpha$ and $m$ dependence has now been included explicitly in the notation. Consider the "slice"

$$
\widetilde{\mathcal{A}}_{\alpha}^{L, m}=\bigcup_{k \leq r} M_{i}^{k \pm} \bigcup_{k \leq r} M_{s}^{k \pm} \cup M_{h} \cup \mathcal{C},
$$

where $r=r_{m}(L)$ and where in $\mathcal{C}$ we have lumped all the connections between all the equilibria $\left\{M_{s}^{k \pm}, M_{i}^{k \pm}, M_{h}\right\}, k \leq r$. We have the following lemma.

Lemma 7.5. In the metastable case, for $\alpha$ sufficiently close to $1, \widetilde{\mathcal{A}}_{\alpha}^{L, m}$ is an isolated invariant set.

Proof. From Theorem 4.10, $\widetilde{\mathcal{A}}_{\alpha}^{L, m}$ will fail to be an isolated invariant set only if an equilibrium $M_{i}^{p \pm}, r<p \leq n$, is arbitrarily close to an element of $\widetilde{\mathcal{A}}_{\alpha}^{L, m}$. However, this would mean that there exists a solution connecting $M_{s}^{k \pm}$, for some $k \leq r$, to $M_{i}^{p \pm}$. Since $p>k$, this would contradict Theorem 1.4.

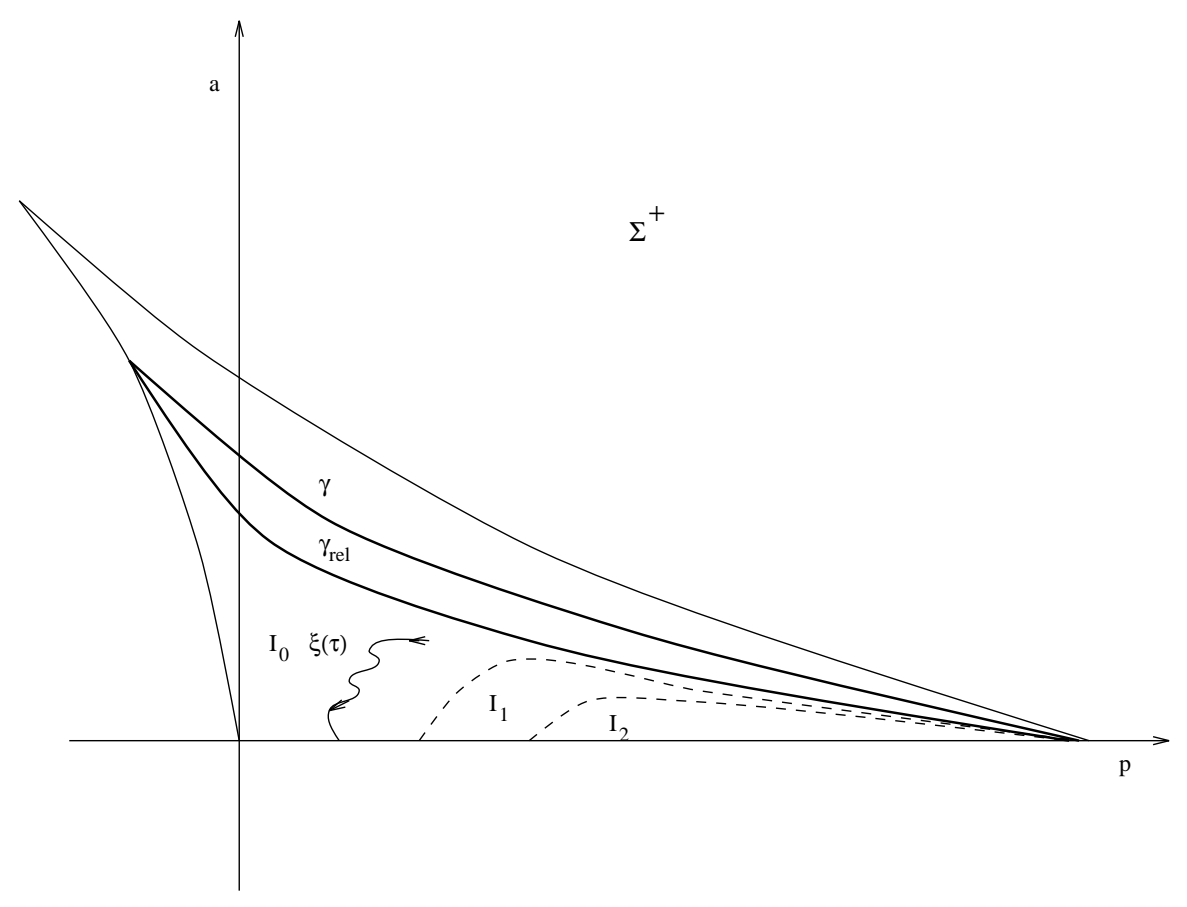

Figure 2. The sets $I_{k}$ 
With regard to the structure of $\widetilde{\mathcal{A}}_{\alpha}^{L, m}$, here is our principal result:

Theorem 7.6. In the metastable case for $\alpha$ sufficiently close to 1, the following connections exist in $\widetilde{\mathcal{A}}_{\alpha}^{L, m}$ :

1. between each $M_{i}^{k \pm}$ and each $M_{i}^{p \pm}, 1 \leq p<k \leq r$;

2. between each $M_{s}^{k \pm}$ and each $M_{s}^{p \pm}, 1 \leq p<k \leq r$;

3. between each $M_{s}^{k \pm}$ and $M_{h}, 1 \leq k \leq r$, and, finally,

4. between each $M_{s}^{k+}$ and each $M_{i}^{p+}, 1 \leq p \leq k \leq r$, with a similar statement holding for the "minus" solutions.

Proof. Step I. Decompose $\widetilde{\mathcal{A}}_{\alpha}^{L, m}$ as follows:

$$
\begin{gathered}
Q_{1}=\bigcup_{k=1}^{r} M_{s}^{k \pm} \cup M_{h} \cup\{\text { all connections among these equilibria }\}, \\
Q_{2}=\bigcup_{k=1}^{r} M_{i}^{k \pm} \cup\{\text { all connections among these equilibria }\},
\end{gathered}
$$

and $\widetilde{\mathcal{A}}_{\alpha}^{L, m}=Q_{1} \cup Q_{2} \cup \mathcal{C}\left(Q_{1}, Q_{2}\right)$. Then we have

Lemma 7.7. $Q_{1}$ is an isolated invariant set and $h\left(Q_{1}\right)=\Sigma^{r}$.

Proof. Let $\left(Q_{1}, Q_{2}\right)$ be the decomposition of $\widetilde{\mathcal{A}}_{\alpha}^{L, m}$ given above. By Lemma 7.4, there exists a continuous curve $\xi(\tau), \tau \in[0,1]$, lying entirely in $I_{r}$, such that $\xi(0)=$ $(a, p)$ corresponds to $(L, m)$ and $\xi(1)$ lies on $\sigma_{0}$, i.e corresponds to $m=0$. The proof now is by continuation in the parameter $\tau$ along $\xi(\tau)$. Note that $\left(Q_{2}(\tau), Q_{1}(\tau)\right)$ is an attractor-repellor pair for $\widetilde{\mathcal{A}}_{\alpha}^{L, m}(\tau)$ for all $\tau \in[0,1]$ and $Q_{2}(\tau)$ is an isolated invariant set. This is the consequence of the fact that the energies of all interface solutions in $\widetilde{\mathcal{A}}_{\alpha}^{L, m}$ are below that of $M_{h}$. This means that $Q_{1}(\tau)$ is an isolated invariant set for all $\tau$. Furthermore, as $h\left(\widetilde{\mathcal{A}}_{\alpha}^{L, m}(\tau)\right)$ and $h\left(Q_{2}(\tau)\right)$ are independent of $\tau$, it follows that $h\left(Q_{1}(0)\right)=h\left(Q_{1}(1)\right)$. However, for $\tau=1$, as we are in $I_{r}$ and $m=0$ we have that $Q_{1}(1)=M_{h}$ and $\tilde{L}^{r}<L<\tilde{L}^{r+1}$; hence $h\left(Q_{1}(1)\right)=\Sigma^{r}$.

Next consider the following Morse decomposition of $\widetilde{\mathcal{A}}_{\alpha}^{L, m}$ :

$$
\widetilde{\mathcal{A}}_{\alpha}^{L, m}=\left\{M_{i}^{1 \pm}, \ldots, M_{i}^{r \pm}, Q_{1}\right\} .
$$

This construction is equivalent to collapsing the whole set $Q_{1}$ to a point. We use the above continuation and the formalism of transition systems [45] to establish all the degree -1 connections in part 1 of the theorem. Note that at $\tau=1$ the connection matrix which we denote by $\Delta_{1}$ (using singular homology with coefficients in $\mathbf{Z}_{2}$ ) is [35] (see the discussion following Theorem 7.1)

$$
\Delta_{1}=\left[\begin{array}{cccc}
O_{2 \times 2} & D & \cdots & \cdots \\
\cdots & O_{2 \times 2} & D & \cdots \\
\cdots & \cdots & \cdots & \cdots \\
\cdots & \cdots & \cdots & O_{2 \times 2}
\end{array}\right]
$$

where the $D$ blocks, which correspond to connections from $M_{i}^{k \pm}$ solutions to $M_{i}^{(k-1) \pm}$ solutions, have the form

$$
D=\left[\begin{array}{ll}
1 & 1 \\
1 & 1
\end{array}\right] .
$$


If $T^{01}$ is the transition matrix from $\tau=0$ to $\tau=1$, then a brute force computation shows that it has to be an identity matrix. Hence the connection matrix at $\tau=0$ which we denote by $\Delta_{0}$ has the same structure as $\Delta_{1}$; this establishes all the degree -1 connections in part $\mathbf{1}$.

Step II. Now consider the finest Morse decomposition of $\widetilde{\mathcal{A}}_{\alpha}^{L, m}$ given by all the Morse sets $M_{i}, M_{s}, M_{h}$. "Reflating" $Q_{1}$ has no influence on the connections established in Step I. Furthermore, the weak lap number principle shows that the Morse sets $M_{i}^{k \pm}$ and $M_{s}^{k \pm}$ are adjacent under the flow defined order. These two facts contain enough information to enable us to determine the missing entries from the connection matrix for this Morse decomposition, as we show next.

Consider the connection matrix $\Delta_{f}$ for this fine Morse decomposition. First, we have that this is a $(4 r+1) \times(4 r+1)$ matrix of maps on homology by counting the equilibria. Its rank is $2 r$ (by the rank condition), and it has the following structure:

$$
\Delta_{\tau}=\left[\begin{array}{ccc}
\Delta_{i i} & O_{2 r \times 1} & \Delta_{s i} \\
O_{1 \times 2 r} & O_{1 \times 1} & \Delta_{s h} \\
O_{2 r \times 2 r} & O_{2 r \times 1} & \Delta_{s s}
\end{array}\right] .
$$

Here $\Delta_{i i}$ is the connection matrix for the interval of interface solutions, which was constructed in step I above; that is, $\Delta_{i i}$ is the submatrix created from the first $2 r$ rows and columns of $\Delta_{0} . \Delta_{s h}$ is a $1 \times 2 r$ matrix and $\Delta_{s s}$ is an $2 r \times 2 r$ matrix. First we compute the matrix $\Delta_{s i}$, which has the structure

$$
\Delta_{s i}=\left[\begin{array}{ccc}
B_{1} & O_{2 \times 2} & \cdots \\
O_{2 \times 2} & B_{2} & \cdots \\
\cdots & \cdots & \cdots
\end{array}\right]
$$

where all $B_{i}$ are $2 \times 2$ matrices. Note that by the weak lap number principle, the set $M_{s}^{k+} \cup M_{i}^{k+} \cup C\left(M_{s}^{k+}, M_{i}^{k+}\right)$ is an isolated invariant set for all $L>\widetilde{L}_{k}$. A connection between these two Morse sets close to that turning point has been established in Lemma 6.4 and the remark following it. Continuing in the parameter $L$ beyond $L=\widetilde{L}_{k}$ we see that the connection persists (again the transition matrix is the identity matrix [45]). Therefore all the blocks are the same, and are just the $2 \times 2$ identity matrices. This proves part 4 of the theorem dealing with degree -1 connections.

Step III. It remains to establish the connections in parts $\mathbf{2}$ and $\mathbf{3}$ of the theorem. There is more than one way of doing that, but probably the easiest one is to remember that $\Delta_{f}$ is a connection matrix, so that $\Delta_{f} \circ \Delta_{f}=O_{(4 r+1) \times(4 r+1)}$. Incorporating the information obtained in Steps I and II, we have that

$$
\Delta_{s s}=\left[\begin{array}{cccc}
O_{2 \times 2} & C & \cdots & \cdots \\
\cdots & O_{2 \times 2} & C & \cdots \\
\cdots & \cdots & \cdots & \cdots \\
\cdots & \cdots & \cdots & O_{2 \times 2}
\end{array}\right]
$$

where the $2 \times 2$ blocks $C$ are matrices of isomorphisms. This proves part $\mathbf{2}$ of the theorem for degree -1 connections. The same computation shows that

$$
\Delta_{s h}=\left[\begin{array}{llll}
1 & 1 & 0 & \cdots
\end{array}\right]
$$

which gives us all the degree -1 connections in part 3 .

All the degree $-n, n>1$ connections in the theorem are then established by appealing to Theorem $\mathrm{E}$ of [30]. 
Denote by $D_{a}^{r}$ the ball in $R^{r}$ of radius $a ; S_{a}^{r-1}=\partial D_{a}^{r}$. We have

Corollary 7.8. The flow on $\mathcal{A}_{L, r}^{\alpha}$ is semiconjugate to the flow $\Psi_{a}^{r}: R \times D_{1}^{r} \rightarrow D_{1}^{r}$ generated by the system of ODEs

$$
\begin{gathered}
\dot{\xi}=Q \xi-\langle Q \xi, \xi\rangle \xi, \quad \xi \in S_{1}^{r-1}, \\
\dot{\rho}=\rho(\rho-a)(1-\rho), \quad \rho \in[0,1], \quad 0<a<1,
\end{gathered}
$$

where $Q$ is an $r \times r$ diagonal matrix, $Q_{i i}=1 / i$ (compare with [35]).

Proof. First of all note that since (see Step I in the proof of Theorem 7.6) all the assumptions A1-A4 of [35] are satisfied for the flow of $\widehat{\mathcal{A}}_{\alpha}^{L, m}$ by which we denote $\widetilde{\mathcal{A}}_{\alpha}^{L, m}$ with the Morse decomposition $\left\{M_{i}^{1 \pm}, \ldots, M_{i}^{r \pm}, Q_{1}\right\}$, we have that that flow is semiconjugate to a Chaffee-Infante flow. Second, if we reverse the direction of the flow on $Q_{1}$, we see that again all the assumptions of the theorem of Mischaikow are satisfied. Therefore it only remains to prove the semiconjugacy of the flow on $\widetilde{\mathcal{A}}_{\alpha}^{L, m} \backslash Q_{1}$ to the flow generated by (7.4)-(7.5) on $\operatorname{int}\left(D_{1}^{r}\right) \backslash D_{a}^{r}$. Assume that there exists a solution curve $\chi(a)$ connecting a rest point on $S_{a}^{r-1}$ to a rest point on $S_{1}^{r-1}$ under $\Psi_{a}^{r}$. Deforming the flow $\Psi_{a}^{r}$ by taking $a$ to 0 , we deform $\chi(a)$ to a trajectory $\chi(0)$ connecting the origin to a rest point on $S_{1}^{r-1}$. Clearly, $\Psi_{0}^{r}$ is conjugate to a Chaffee-Infante flow [35], so the flow on $\widehat{\mathcal{A}}_{\alpha}^{L, m}$ is semiconjugate to $\Psi_{0}^{r}$. Therefore $\chi(0)$ corresponds to a trajectory on $\widehat{\mathcal{A}}_{\alpha}^{L, m}$. But then by the proof of Theorem 7.6, $\chi(a)$ corresponds to a trajectory on $\widetilde{\mathcal{A}}_{\alpha}^{L, m}$.

\section{Remarks AND CONCLUSiOnS}

In this paper we have added to the collection of bifurcation diagrams obtained in [27] the related information concerning the energy levels of all the stationary states of the viscous Cahn-Hilliard homotopy. We have further demonstrated a joint continuity property for the set of global attractors in terms of mean mass and the homotopy parameter $\alpha$. Hyperbolicity of the set of equilibria has been demonstrated away from turning points and points of bifurcation from the homogeneous state, and combined with local stability analysis, the index of instability is calculated for all equilibria. Combining the above information, partial information is obtained on the possible connections which may exist on the global attractor.

Here we would like to comment on the difficulties associated with any further analysis of the attractor of the viscous Cahn-Hilliard homotopy. One difficulty is technical; i.e., it is easy to check that it is possible to find values of the parameters $L$ and $m$ so that the energy of some spike solutions will be lower than that of some interface solutions. Thus even though the energy of all the stationary states are calculable, it may be cumbersome to prescribe further, more detailed Morse decompositions.

Another problem is the fact that the energy-defined Morse decomposition of the attractor in the transitional and metastable cases does not determine the connection matrix uniquely. In such a case, additional information on the dynamical system is required to be able to fix the structure of connections. An elegant theory of connections for scalar reaction-diffusion equations in one dimension can be found in $[19,20]$. We note that these authors do not use energy information, but instead rely on consequences of the maximum principle, which provide a very precise description of stable and unstable manifolds of equilibria. In particular, it is 
possible to prove $[5,32]$ that the flows generated by such dynamical systems are Morse-Smale. This additional information allows one to rule out certain topologies of attractors. The question whether the viscous Cahn-Hilliard equation generates a Morse-Smale dynamical system is thus of considerable interest. It is clear that

if the answer is positive, energy information obtained in this paper will uniquely determine the pattern of connections.

It would also be of interest to understand whether the weak lap number principle holds for all values of $\alpha \in[0,1]$, as this would allow us to prove the equivalent of Theorem 7.6 for the whole homotopy.

Lastly, there may be other gradient systems or perturbations of gradient systems for which the structure of the global attractor might be accessible with the methods developed here.

\section{ACKNOWLEDGMENT}

M. G. would like to thank Professor K. Mischaikow for valuable discussions. A. N.-C. would like to acknowledge the support by the fund for the Promotion of Research at the Technion.

\section{REFERENCES}

1. N. Alikakos, P.W. Bates and G. Fusco, Slow motion for Cahn-Hilliard equation in one space dimension, J. Diff. Equations, 90 (1991), 81-134. MR 92a:35152

2. S. Allen and J.W. Cahn, A microscopic theory for antiphase boundary motion and its application to antiphase domain coarsening, Acta Metall. 27 (1979), 1084-1095.

3. N. Alikakos and G. Fusco, Equilibrium and dynamics of bubbles for the Cahn-Hilliard equation, Collection: Inter. Conf. on Differential Equations, vol 1,2 (Barcelona, 1991), 59-67, World Sci. Publish., River Edge, NJ (1993). MR 95b:35111

4. N. Alikakos and G. Fusco, Slow dynamics for the Cahn-Hilliard equation in higher space dimensions. Part I: Spectral estimates, Comm. in PDEs 19 (1994), 1397-1447. MR 95j:35163

5. S. B. Angenent, The Morse-Smale property for a semilinear parabolic equation, JDE 62 (1986), 427-442. MR 87e:58115

6. F. Bai, C. M. Elliott, A. Gardiner, A. Spence and A. M. Stuart, The viscous Cahn-Hilliard equation. Part I: Computations, Nonlinearity 8 (1995), 131-160. MR 95m:35082

7. P. W. Bates and P. C. Fife, Spectral comparison principles for the Cahn-Hilliard and phase field equations, and time scales for coarsening, Physica 43D (1990), 335-348. MR 91h:80001

8. P. W. Bates and P. C. Fife, The dynamics of nucleation for the Cahn-Hilliard equation, SIAM J. Appl. Math, 53 (1993), 990-1008. MR 94g:82034

9. P. W. Bates and P. J. Xun, Metastable patterns for the Cahn-Hilliard equation, Part II, J. Diff. Equations, 117, (1995), 165-216. MR 95m:35188

10. D. Brochet, D. Hilhorst, and A. Novick-Cohen, Maximal attractor and inertial sets for a conserved phase field model, Advances in PDE 1(1996), 547-578. MR 97e:35189

11. L. Bronsard and D. Hilhorst, On the slow dynamics for the Cahn-Hilliard equation in one space dimension, Proc. Roy. Soc. London, Series A, 439 (1992), 669-682. MR 93k:35210

12. J.W. Cahn and J.E. Hilliard, Free energy of a nonuniform system. I. Interfacial free energy, J. Chem. Phys. 28 (1958), 258-267.

13. J. Carr, M. E. Gurtin and M. Slemrod, Structured phase transitions on a finite interval, Arch. Rat. Mech. Anal. 86 (1984), 317-354. MR 86i:80001

14. C. C. Conley, Isolated Invariant Sets and Morse Index, AMS Regional Conference Series in Mathematics 38 AMS, Providence, RI (1978). MR 80c:58009

15. A. Eden, C. Foias, B. Nicolaenko, and R. Temam, Inertial sets for dissipative evolution equations. Part I: Construction and application, IMA Preprint 812 (1991).

16. J. C. Eilbeck, J. E. Furter and M. Grinfeld, On a stationary state characterization of transition from spinodal decomposition to nucleation behavior in the Cahn-Hilliard model of phase separation, Phys. Lett. A 139 (1989), 42-46. MR 89k:80015 
17. C. M. Elliott A. M. Stuart, The viscous Cahn-Hilliard equation. Part II: Analysis, J. Diff. Equations 128 (1996), 387-414. MR 97c:35080

18. C. M. Elliott and S. Zheng, On the Cahn-Hilliard equation, Arch. Rational Mech. Anal. 96 (1986), 339-357. MR 87k:80007

19. B. Fiedler, Global attractors of one-dimensional parabolic equations: 16 examples, Tatra Mount. Math. Publ. 4 (1994), 67-92. MR 95g:35089

20. B. Fiedler and C. Rocha, Heteroclinic orbits of semilinear parabolic equations, J. Diff. Equations 125, (1996) 239-281. MR 96k:58200

21. P.C. Fife, Models for phase separation and their mathematics, in Nonlinear Partial Differential Equations and Applications, M. Mimura and T. Nishida, eds., Kinokuniya Pubs., Tokyo, in press.

22. R. D. Franzosa, The continuation theory for Morse decompositions and connection matrices, Trans. AMS 310 (1988), 781-803. MR 90g:58111

23. R. D. Franzosa, The connection matrix theory for Morse decompositions, Trans. AMS 311 (1989), 561-592. MR 90a:58149

24. P. Freitas, Stability of stationary solutions for a nonlocal reaction-diffusion equation, Quart. J. Mech. Appl. Math., 48 (1995), 556-582. MR 97a:35111

25. G. Fusco and J.K. Hale, Slow motion manifolds, dormant instability and singular perturbations, Dynamics Differential Equations 1 (1989), 75-94. MR 90i:35131

26. M. Grinfeld, J. E. Furter and J. C. Eilbeck, A monotonicity theorem and its applications to stationary solutions of the phase field model, IMA J. Appl. Math. 49 (1992), 61-72; 50 (1993), 203-204. MR 93j:80003

27. M. Grinfeld and A. Novick-Cohen, Counting stationary solutions of the Cahn-Hilliard equation by transversality arguments, Proc. Royal Soc. Edin. A 125 (1995), 351-370. MR 96c: 58157

28. M. E. Gurtin and H. Matano, On the structure of equilibrium phase transitions within the gradient theory of fluids, Quart. Appl. Math. 46 (1988), 301-317. MR 89j:49015

29. J. K. Hale, Asymptotic Behaviour of Dissipative Systems, Math. Surveys and Monographs 25 A.M.S. 1988. MR 89g:58059

30. H. Hattori and K. Mischaikow, A dynamical system approach to a phase transition problem, J. Diff. Equations 94 (1991), 340-378. MR 93m:58104

31. J. K. Hale and G. Raugel, Lower semicontinuity of attractors of gradient systems and applications, Ann. di Matem. Pura ed Applic. 154 (1989), 281-326. MR 91f:58087

32. D. Henry, Some infinite-dimensional Morse-Smale systems defined by parabolic differential equations, J. Diff. Equations 59 (1985), 165-205. MR 86m:58080

33. J. Mallet-Paret, Morse decompositions for delay-differential equations, J. Diff. Equations $\mathbf{7 2}$ (1988), 270-315. MR 80m:58112

34. H. Matano, Nonincrease of the lap number of a solution for a one-dimensional semilinear parabolic equation, J. Fac. Sci. Univ. Tokyo Sect. 1A Math. 29 (1982), 401-441. MR 84m:35060

35. K. Mischaikow, Global asymptotic dynamics of gradient-like bistable equations, SIAM J. Math. Analysis 26 (1995), 1199-1224. MR 96j:35025

36. B. Neithammer, Existence and uniqueness of radially symmetric stationary points within the gradient theory of phase transitions, Eur. J. Appl. Math. 6 (1995), 45-68.

37. B. Nicolaenko, B. Scheurer and R. Temam, Some global dynamical properties of a class of pattern formation equations, Comm. PDE 14 (1989), 245-297. MR 90e:35138

38. A. Novick-Cohen, The nonlinear Cahn-Hilliard equation: transition from spinodal decomposition to nucleation behavior, J. Stat. Phys. 38 (1985), 707-723.

39. A. Novick-Cohen, On the viscous Cahn-Hilliard equation, in: Material Instabilities in Continuum Mechanics and Related Mathematical Problems, J. M. Ball, ed., Oxford University Press, Oxford 1988. MR 89f:73005

40. A. Novick-Cohen, On Cahn-Hilliard type equations, Nonlinear Analysis TMA 15 (1990), 797-814. MR 92g:35105

41. A. Novick-Cohen, The Cahn-Hilliard equation: mathematical and modelling perspectives, Advances in Math. Sci. and Appl. 8 (1998), 965-985.

42. A. Novick-Cohen and L. A. Peletier, Steady states of the one-dimensional Cahn-Hilliard equation, Proc. Royal Soc. Edin. A 123 (1993), 1071-1098. MR 94m:35272

43. A. Novick-Cohen and L. A. Segel, Nonlinear aspects of the Cahn-Hilliard equation, Physica 10D (1984), 277-298. MR 85k:35120 
44. I. Ohnishi and Y. Nishiura, Spectral comparison between the second and fourth order equations of conservative type with nonlocal terms, Japan J. Indust. Appl. Math. 15 (1998), $253-262$.

45. J. Reineck, Connecting orbits in one-parameter families of flows, Erg. Theory Dynam. Sys. 8* (1988), 359-374. MR 89i:58128

46. J. Rubinstein and P. Sternberg, Non-local reaction-diffusion equations and nucleation, IMA J. Appl. Math. 48 (1992), 249-264. MR 93f:35116

47. R. Schaaf, Global Solution Branches of Two-Point Boundary Value Problems, Lecture Notes in Mathematics 1458, Springer-Verlag, Berlin 1990. MR 92a:34003

48. P. Sternberg and K. Zumbrun, Connectivity in phase boundaries in strictly convex domains, Arch. Rat. Mech. Anal. 141 (1998), 375-400.

49. R. Temam, Infinite-Dimensional Dynamical Systems in Mechanics and Physics, Applied Math. Sciences 68, Springer-Verlag, New York, 1988. MR 89m:35204

50. J. Wei and M. Winter, Stationary solutions for the Cahn-Hilliard equation, Ann. Inst. H. Poicare, Anal. Non Lin., 15 (1998), 459-492. CMP 98:15

51. S. Zheng, Asymptotic behaviour of solutions to the Cahn-Hilliard equation, Applic. Analysis 23 (1986), 165-184. MR 88b:35036

Department of Mathematics, University of Strathclyde, Livingstone Tower, 26 Richmond Street, Glasgow G1 1XH, Scotland, United Kingdom

E-mail address: michael@conley.maths.strath.ac.uk

Faculty of Mathematics, Technion-IIt, Haifa 32000, Israel

E-mail address: amync@techunix.technion.ac.il 